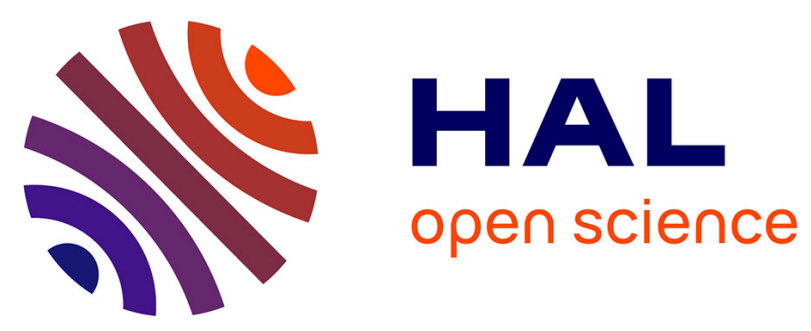

\title{
Managing the challenge of drug-induced liver injury a roadmap for the development and deployment of preclinical predictive models
}

Christopher E Goldring, André Guillouzo, Philip G Hewitt, Magnus Ingelman-Sundberg, Klaus Gjervig Jensen, Satu Juhila, Ursula Klingmüller, Gilles Labbe, Michael J Liguori, Cerys A Lovatt, et al.

\section{To cite this version:}

Christopher E Goldring, André Guillouzo, Philip G Hewitt, Magnus Ingelman-Sundberg, Klaus Gjervig Jensen, et al.. Managing the challenge of drug-induced liver injury a roadmap for the development and deployment of preclinical predictive models. Nature Reviews Drug Discovery, 2020, 19

(2), pp.131-148. 10.1038/s41573-019-0048-x . hal-02397617

\section{HAL Id: hal-02397617}

\section{https://hal-univ-rennes1.archives-ouvertes.fr/hal-02397617}

Submitted on 16 Jun 2021

HAL is a multi-disciplinary open access archive for the deposit and dissemination of scientific research documents, whether they are published or not. The documents may come from teaching and research institutions in France or abroad, or from public or private research centers.
L'archive ouverte pluridisciplinaire HAL, est destinée au dépôt et à la diffusion de documents scientifiques de niveau recherche, publiés ou non, émanant des établissements d'enseignement et de recherche français ou étrangers, des laboratoires publics ou privés. 


\section{Managing the Challenge of Drug Induced Liver Injury: A roadmap for the development and deployment of preclinical predictive models}

\section{AUTHORS}

Richard J Weaver ${ }^{1}$, Eric A Blomme ${ }^{2}$, Amy E Chadwick ${ }^{3}$, lan M Copple ${ }^{3}$, Helga HJ Gerets ${ }^{4}$, Christopher E Goldring ${ }^{3}$, Andre Guillouzo ${ }^{5}$ Philip G Hewitt ${ }^{6}$, Magnus Ingelman-Sundberg ${ }^{7}$, Klaus Gjervig Jensen ${ }^{8}$, Satu Juhila ${ }^{9}$, Ursula Klingmüller ${ }^{10}$, Gilles Labbe ${ }^{11}$, Michael J Liguori ${ }^{2}$, Cerys A Lovatt ${ }^{12}$, Paul Morgan ${ }^{13}$, Dean J Naisbitt ${ }^{3}$, Raymond HH Pieters ${ }^{14}$, Jan Snoeys ${ }^{15}$, Bob van de Water ${ }^{16}$, Dominic P Williams ${ }^{13}$, B Kevin Park ${ }^{3}$

1. Institute de Recherches Internationales Servier, Biopharmacy, rue Carnot, 92284, Suresnes, France

2. Abbvie, Global Preclinical Safety, 1 North Waukegan Road, R46G/AP13A-3, North Chicago, IL 60064

3. MRC Center for Drug Safety Science, Department of Molecular and Clinical Pharmacology, University of Liverpool, Sherrington Building, Ashton Street, Liverpool , L69 3GE , England.

4. UCB BioPharma SPRL, Developmental Sciences, Chemin du Foriest, 1420 Braine-l'Alleud, Belgium

5. University Rennes, Inserm, Inra, Institut NUMECAN (Nutrition Metabolisms and Cancer), UMR_S 1241, 35000 Rennes, France

6. Merck KGaA, Non-Clinical Safety, Darmstadt, Germany

7. Karolinska Institutet, Department of Physiology and Pharmacology, Biomedicum 5B, Stockholm, Sweden

8. H Lundbeck, Drug ADME Research, H. Lundbeck A/S, 9 Ottiliavej, Valby, Denmark.

9. Orion Pharma, In Vitro Biology, Espoo, Finland

10. Deutsches Krebsforschungszentrum (DKFZ), Systems Biology of Signal Transduction, German Cancer Research Center, Heidelberg, Germany

11. Sanofi, Investigative Toxicology, Preclinical Safety, Sanofi R\&D, Paris, France.

12. GlaxoSmithKline, R\&D, Ware, Hertfordshire, UK

13. AstraZeneca, Drug Safety and Metabolism, IMED Biotech Unit, Cambridge Science Park, Cambridge, CB4 OWG, UK.

14. Institute for Risk Assessment Sciences, Immunotoxicology, Faculty of Veterinary Medicines, Utrecht University, Utrecht, The Netherlands

15. Drug Metabolism and Pharmacokinetics, Janssen Research and Development, Turnhoutseweg 30, Beerse, Belgium

16. Universiteit Leiden, Division of Drug Discovery and Safety, Leiden Academic Centre for Drug Research, Leiden University, Einsteinweg 55, 2333 CC, Leiden, The Netherlands 


\section{Authors for Correspondence}

B Kevin Park

MRC Centre for Drug Safety Science, Department of Molecular and Clinical Pharmacology, University of Liverpool, Sherrington Building, Ashton Street, Liverpool, L69 3GE, England.

Richard J Weaver

Institute de Recherches Internationales Servier, Biopharmacy, rue Carnot, 92284, Suresnes, France 


\section{Glossary}

2D, 2-dimensional; 3D, 3-dimensional; ADME, Absorption, Distribution, Metabolism Excretion; ADR, Adverse Drug Reaction; ADRs, Adverse Drug Reactions; AE, Adverse event; AEs, Adverse events; APAP, acetaminophen; ATF6, Activating transcription factor 6; ATP, adenosine triphosphate; BA, bile acid; BAC, bacterial artificial chromosome; $B C$, bile canaliculi; $B C R P$, breast cancer resistance protein; Bip, Binding immunoglobulin protein; BSEP, bile salt export pump; $C S A$, cyclosporine $A ; C A$, cholic acid; CADs, Cationic amphiphilic drugs; CDCA, chenodeoxycholate; CHOP, C/EBP homologous protein; ClogP, The logP value of a compound; Cmax, maximum concentration; CPT-1, carnitine palmitoyltransferase-1; CRM, chemically reactive metabolites; Css, Concentration at Steady State; CTLA-4, cytotoxic T-lymphocyte-associated protein 4; CYP, cytochrome P450; DAMPs, damageassociated molecular patterns; DCA, deoxycholate; DIC, diclofenac; DILI, drug-induced liver injury; DILI-sym, drug-induced liver injury modelling software; EFPIA, European Federation of Pharmaceutical Industries and Associations; ENT, equilibrative nucleoside transporter; ER, endoplasmic reticulum; GCDCA, glycochenodeoxycholate; GFP, green fluorescent protein; Glu-Gal, glucose-galactose; GSH, glutathione; HCA, high-content analysis; hENT1, human endonucleoside transporter-1; HLA, human leukocyte antigen; HMGB1, high mobility group box protein-1; ICAM1, intercellular Adhesion Molecule 1; IFN- $\gamma$, interferon- $\gamma$; IL-1 $\beta$, interleukin-1 $\beta$; IL-8, interleukin-8; iPS, induced pluripotent stem cells; IRE1 $\alpha$, inositol-requiring enzyme $1 \alpha$; IVIVE, in vitro-in vivo; LCA, lithocholic acid; LPS, lipopolysaccharide; LSEC, liver sinusoidal endothelial cell; luc, luciferase; MATE, Multidrug and toxin extrusion protein; MICS, microphysiological systems; MIP-DILI, MechanismBased Integrated Systems for the Prediction of Drug-Induced Liver Injury; MLC, myosin light chain; MLCK, myosin light chain kinase; MRP, multidrug resistance-associated protein; mtDNA, mitochondrial DNA; NCE, new chemical entity; NCEs, new chemical entities; NFkB, nuclear factorkappa B; NK, natural killer; NPCs, non-parenchymal cells; Nrf2, nuclear factor erythroid 2-related factor 2; NTCP, Na+-taurocholate co-transporting polypeptide; OATP, organic anion transporting polypeptide; OAT, Organic Anion Transporter; OCT, organic cation transporter; OST, Organic solute transporter; PBMC, peripheral blood mononuclear cells; PD1, Programmed cell death protein 1; PERK, protein kinase RNA-like endoplasmic reticulum kinase; PHH, primary human hepatocyte; PKPD, Pharmacokinetics \& pharmacodynamics; PL, phospholipidosis ; R\&D, research and development; RelA, REL-associated protein; ROCK, Rho kinase; ROS, reactive oxygen species; S100A9, S100 calcium-binding protein A9; SCHH, sandwich-cultured human hepatocyte; SOS, sinusoidal obstructive syndrome; SRXN1, sulfiredoxin 1; TCA, taurocholic acid; TCR, T-cell receptor; TK-NOG, thymidine kinase transgene incorporated into immunodeficient NOG mice; TNF- $\alpha$, tumor necrosis factor- $\alpha$; TRAF2, TNF receptor-associated factor 2; Tregs, regulatory T cells; VBDS, vanishing bile duct syndrome; UPR, unfolded protein response; XBP1, X-box binding protein 1. 


\section{Abstract}

Drug-induced liver injury (DILI) is a patient-specific, temporal, multifactorial pathophysiological process that cannot yet be recapitulated in a single in vitro model. Current pre-clinical testing regimes for the detection of human DILI thus remain inadequate. A systematic and concerted research effort is required to address the deficiencies in current models and to present a defined approach towards the development of new or adapted model systems for DILI prediction. This Perspective defines the current status of available models and mechanistic understanding of DILI, and proposes our vision of a roadmap for the development of predictive preclinical models of human DILI. 


\section{Introduction}

Adverse drug reactions (ADRs) are a major clinical problem in terms of patient morbidity, patient mortality, cost to healthcare systems and failure of drugs in development. The liver is one of the organs most susceptible to drug toxicity, and in the clinic, drug-induced liver injury (DILI) has accounted for more than $50 \%$ of acute liver failure cases ${ }^{1}$. Excluding acetaminophen, DILI accounts for approximately $14 \%$ of acute liver failures with a mortality rate of up to $10 \%^{2}$. DILI is also a major cause of drug attrition, leading to withdrawal of potentially valuable therapies, both during preclinical testing, clinical trials and post-marketing ${ }^{3,4}$. Importantly, the FDA has annotated 750 drugs that have some degree of DILI risk ${ }^{5}$. However, it is clear that current preclinical testing paradigms based on a combination of various in vitro and in vivo models are poorly predictive, at a quantitative and mechanistic level, of the potential of a new drug candidate to cause DILI in humans, in particular those drugs that show poorly defined dose-response relationships and/or human specific mechanisms of toxicity.

There is an emerging body of evidence that DILI, as it occurs in humans, can be a multi-step and multicellular disease process with a diverse range of chemical aetiologies (Figure 1$)^{6-8}$. This means that prediction of all forms of DILI may be inherently intractable to simple solutions, such as single cell culture screening strategies. Thus, a much better understanding of the mechanisms underlying DILI is essential in order to a) evaluate the strengths and weaknesses of currently available test systems and b) inform the design and construction of new improved predictive models. Crucially, we must define not only whether a test system is fit-for-purpose, but more critically "what particular purpose is a test system fit for". In addition, we need to be aware of which forms of DILI we can predict and which forms of DILI we cannot predict, when there is no human experience with the drug.

Given the multifactorial mechanisms of DILI, which contribute to drug attrition in development and in clinical practice, there is a need for new thinking in terms of the development of a holistic approach to the early detection of chemical liabilities which are predictive of DILI. Such an approach must be

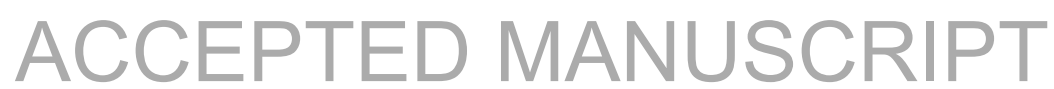


mechanism-based, pragmatic and sufficiently adaptable to be of practical application to 1) influence drug design early enough in the discovery phase and 2) manage risk assessment in drug development, which is amenable to evaluation by drug regulators, but cognisant of current limitations in our understanding of DILI. This is particularly important when human risk factors dominate over chemical risk factors, e.g. for idiosyncratic drug toxicity.

It is clear from a review of the literature, that no single system is fit-for-purpose as a universal test for DILI in humans, which is a patient-specific, temporal, multifactorial pathophysiological process. Therefore, what is required is a portfolio of robust and well-characterised predictive DILI platforms that have their purpose well-defined, and acceptable in a theoretical and practical sense to academic, industry and regulatory agencies.

This perspective presents our vision for a DILI roadmap, with the aim of managing DILI risk and ultimately assisting in the design and development of safer and more effective medicines. Our approach is comprised of a tiered system, which integrates established and emerging cell-based technologies into a coherent map for drug development and, ultimately, for drug regulation. 


\section{Proposed DILI Roadmap}

Potential strategies to de-risk DILI in drug discovery have previously been published. These approaches, whether presented as in vitro hazard/liability matrices ${ }^{9,10}$ or tiered testing cascades, are typically those of a single institution or focus only on single mechanisms, proprietary test systems ${ }^{11-}$ ${ }^{14}$, legacy compound datasets ${ }^{15}$, and/or technology platforms ${ }^{16,17}$. For this reason, there has remained little or no consensus on the use or approach to de-risk DILI. Moreover, many of these strategies may not be readily amenable for adoption by small enterprises and start-ups where inhouse capacity and availability of proprietary compounds to validate such approaches are lacking.

The DILI Roadmap discussed in this Perspective was established by nine medium-large EFPIA pharmaceutical companies, SMEs and academic partners as a holistic strategy to manage human DILI risk. Our approach delivers an in-depth inter-laboratory evaluation of the fundamental and reproducible performance of assays, to identify chemical liabilities in drug discovery and to manage DILI risk in development. Our roadmap (Figure 2) integrates established and emerging test systems, and is based on a 3-tiered approach, whereby the complexity of the model increases progressively from single cell 2D to multi-cell 3D systems through to systems that incorporate human individualspecific factors, such as genetic- or disease-related factors.

As the Roadmap incorporates assays and cell models which are well-characterized and have proven their utility ${ }^{18-20}$ as well as emerging novel systems with anticipated future benefit, this enables the versatility of the Roadmap to evolve with the advent of future qualified complex DILI models

\section{Cell Models}

There are numerous examples of significant interspecies differences in DILI, caused for example by species variations in the specificity of drug metabolism and exerted toxicity. Accordingly, humanbased in vitro systems for predicting in vivo DILI in man are preferable. The in vitro systems should 
exert a relevant phenotype, mimicking the functionality of hepatocytes in vivo and, since many drugs exert a delayed DILI response, the system must exhibit long-term stability.

Much attention has been placed on the refinement of 2D cell systems and development of a 3D spheroid system based largely on primary hepatocytes, where the transcriptomic, proteomic and metabonomic profile as well as the functionality of the spheroids are very similar to the specific donor liver ${ }^{18,21-23}$. Such spheroids have also been shown to imitate liver disease induced by altered physiological conditions ${ }^{24}$. When challenged in long-term cultures with 122 drugs with or without direct implication in clinical DILI, this spheroid system successfully detected $69 \%$ of all hepatotoxic compounds without producing any false positive results ( $100 \%$ specificity), thereby exceeding all previously published in vitro assays at substantially lower drug concentration levels, that approach Cmax or Css in blood with drugs known to cause human DILI ${ }^{23}$. Single cell models, whether primary or immortalised, nevertheless lack the intricacies of a multicellular environment, and whilst a range of inventive technologies have been developed to build complex 3-D structural models, none published so far has been able to recapitulate the complex physiology of the intact liver.

Much effort is now being directed to develop different kinds of more physiologically-relevant in vitro systems such as $2 \mathrm{D}$ and $3 \mathrm{D}$ multi-cellular tissue chip and microfluidic systems which can provide a relevant cellular milieu for studies of liver function and DILI ${ }^{25,26}$. In addition, microfluidic microphysiological (organ-on-chip) systems are being built in a multi-organ fashion which in the future may be able to further integrate complex mechanisms relevant for the production of idiosyncratic human DILI'

However, despite significant efforts towards developing the next generation of advanced in vitrobased liver models with greater physiological relevance, none are presently in routine use by industry. This in part is due to remaining technological challenges and a required demonstrable 'paradigm shift' in predictive power yet to be established ${ }^{28-30}$. 2D cell-based assays platforms therefore remain 
common practice for screening due to their ease of use, availability and suitability for $\mathrm{HTS}^{28,31}$, despite their reported limitations of physiological relevance ${ }^{8,32}$.

It may not be possible to combine all features of a fully functioning liver in microphysiological tissuebased systems (MPS) models, thereby requiring the integration of input from more than a single test system. Nevertheless, there is an opportunity to capitalize on the use of established $2 \mathrm{D}$ cell models and assays in a tiered and congruent manner that applies physiological and pharmacological knowledge, advantages and limitations of these 2D cell models, with more advanced models as they become available and qualified for use. Cell-based 2D models currently comprise traditional liver derived cell-lines and primary hepatocytes. Features facilitating use of 2D cell models such as HepG2, HepaRG, and primary hepatocytes are summarized elsewhere ${ }^{33}$ and in Table 1 , and represent the most commonly available and widely used cell models by industry. Given this, along with the breath of available literature on the characterization of HepG2 and HepaRG, this prioritizes the use of these cell models in a tiered approach for screening of key mechanisms associated with human DILI.

In addition, the strategy described in this Perspective for assessing DILI risk encompasses the application of cell models most appropriate for a given mechanism being studied (sections 3-8; mitotoxicity, ER stress, or bile canilicular dilatation). Hence, each individual cell model is not only defined as uniquely a TIER 1 or TIER 2 test, but also by the toxicological endpoint(s) under evaluation 


\section{Physicochemical characteristics associated with DILI risk}

High daily doses and high systemic exposures to drugs are associated with increased risk of DILI ${ }^{34,35}$. It is therefore important to account for the intended pharmacological drug exposure (concentration) and ADME data alongside the use of the in vitro DILI roadmap (Figure 2), to help integrate concentration-effect relationships for the prediction of DILI. Low doses are desirable and ideally doses $<100 \mathrm{mg} /$ day should be targeted for oral drugs ${ }^{36}$. For example, evaluation of the top 200 oral drugs in 2009 in the USA and of 68 drugs recalled or associated with a black box warning due to idiosyncratic toxicity, indicated that the major differentiating factor between the two groups of drugs was the total daily dose ${ }^{37}$. Likewise, the vast majority of oral drugs with reported idiosyncratic liver toxicity are administered at high clinical doses ${ }^{38}$.

Not surprisingly, given their influence on efficacious doses, some physicochemical properties (e.g. lipophilicity, polar surface area, number of hydrogen bond donors and acceptors) have also been shown to be associated with an increased risk of DILI. For this reason, determining the physiochemical properties of a compound is important. Computational tools are available as TIER 1 assays to guide early drug design by predicting physicochemical properties or sites of metabolism, metabolite structure, cytochrome P450 (CYP) binding/inhibition, and CYP induction ${ }^{39,40}$. Examples include the Meteor program, MetaSite, and ADMET Predictor ${ }^{39,41,42}$. The links between compound characteristics of interest (e.g., physicochemical properties, bioactivation, and general toxicity) and molecular structure (i.e., 'the similarity principle') form the basis of these in silico applications that differ only in terms of complexity and performance ${ }^{43}$. For example, various toxicophores or problematic substructures can be identified with this approach ${ }^{44,45}$. These computational assessments are typically conducted prior to TIER I assays in order to prevent the synthesis of compounds with low probability of success or to prioritize screening of subsets of compounds, thereby optimizing drug discovery efforts. However, in silico tools still have some limitations and traditional "wet-lab" experimentation with data generation as depicted in the Roadmap as TIER 1 assays is recommended for screening of 
compounds to confirm predicted readouts from these in silico applications (i.e., spot-checking exercise). Several quantitative systems toxicology platforms have been proposed to predict and understand DILI. DILIsym ${ }^{\circledast}$ is an in silico approach to predict DILI based on known mechanisms ${ }^{46}$. The software relies on predetermined mechanisms, such as oxidative stress, glutathione metabolism, mitochondrial dysfunction, and ATP depletion to simulate a DILI outcome ${ }^{41}$. The DILI prediction system (DILIps) uses a quantitative structure-activity relationship (QSAR) approach to classify a compound's propensity to induce DILI ${ }^{47}$ by use of drugs known to cause DILI. Whilst retrospective analyses of known hepatotoxic drugs help to identify potential hazard, quantifying risk and predictive value and identification of some types of DILI, such as idiosyncratic DILI in patients is not yet achievable. The main points concerning the importance of dose and physicochemical properties are presented in BOX 1 and discussed in further detail in the supplementary section.

\section{Intracellular perturbations associated with DILI liability}

A number of intracellular chemical perturbations elicited by pharmaceutical compounds are associated with a DILI liability, including mitochondrial dysfunction, inhibition of biliary efflux, lysosomal impairment, production of reactive metabolites, endoplasmic reticulum stress, as well as involvement of the immune system (Figure 1). Below we will discuss these factors and examine how they can be evaluated using our tiered testing strategy as shown in the DILI roadmap (Figure 2).

\section{Mitochondrial dysfunction}

In a recent study of 300 drugs, $50-60 \%$ of those that can elicit idiosyncratic toxicity cause mitochondrial perturbation, whereas amongst non-DILI drugs this falls to $<5 \%{ }^{48}$, (supplemental section, Figure 1). However, in many cases where hepatotoxicants have been shown to contain mitochondrial liabilities, there is limited clinical evidence linking this mitochondrial dysfunction to the onset of DILI. 
Over the past decade the pharmaceutical industry has sought to incorporate the detection of compounds capable of inducing mitochondrial dysfunction into preclinical testing strategies ${ }^{49}$. The mainstay of such testing stems from the recognition that the use of cells of a tumorigenic nature obscures detection of mitotoxicity. This is due to an altered bioenergetic phenotype which enables them to use glycolysis to produce ATP, not mediated via mitochondrial oxidative phosphorylation, to sustain continued growth and proliferation. To circumvent this, the technique of metabolic modification to identify compounds which contain mitochondrial liabilities was described ${ }^{50,51}$. Specifically, HepG2 cells are adapted to culture in either glucose- or galactose-containing media over a period of weeks and then exposed to compounds for 24 hours. A rightward shift in cell death or ATP dose-response curves in galactose media, due to a complete dependence upon oxidative phosphorylation for ATP generation, provides evidence for mitochondrial dysfunction. However, despite widespread adoption, the glu-gal assay has low prediction rates and subsequent work amended the original protocol ${ }^{52}$. For example, acute exposure (2-8 hours) of HepG2 cells to test compounds and dual assessment of cellular ATP content and cell death in cells in which the metabolic switch is made over $2 \mathrm{~h}^{53,54}$ enables an additional evaluation of whether mitochondrial dysfunction is a cause of cell death ${ }^{53}$. Characterisation of this HepG2 model revealed its utility to detect and rank, in terms of potency, those compounds, parent or synthetic equivalents of any human metabolites, which directly induce mitochondrial dysfunction via interference with the electron transport chain. However, its evaluation using a larger set of compounds is still required to determine whether these changes result in a significant improvement in specificity and selectivity. The simplicity and ease of the acute metabolic switch (glu-gal) assay when used over an acute period, in contrast to other forms of this assay, facilitates its use as a TIER 1 assay; i.e. to be adopted early in the preclinical testing strategy to rapidly screen compounds. The principle of in vitro, metabolic modification is applicable to other methods of screening for mitochondrial dysfunction, in particular mitochondrial membrane potential, respirometry or high content analysis, which can be used as an alternative option to identify compounds with a mitochondrial liability. If a compound is identified as positive, the subsequent

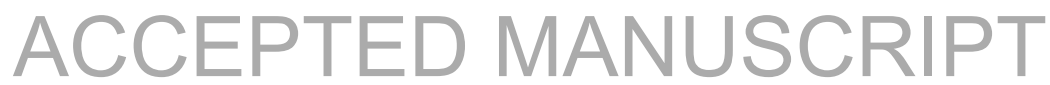


investigation to confirm classification and define mechanisms can include traditional respirometry in isolated mitochondria or the use of a Seahorse extracellular flux analyser for membrane potential. These two assays allow the measurement of both oxidative phosphorylation and glycolytic function in real-time, in whole and permeabilised cells or isolated mitochondria ${ }^{53,55}$.

After an initial screen of hazard identification on a large-scale, the next stage of mitochondrial testing, TIER 2, should take place in more complex models designed to have greater physiological relevance and closer relevance to clinical exposure i.e. over weeks instead of days. This is particularly important as drug-induced mitochondrial dysfunction can often form part of a multi-mechanistic pathway, working in concert with other processes within the hepatocyte, such as biliary transport and xenobiotic metabolism ${ }^{49}$. Furthermore, mitochondrial toxicity often has a delayed presentation over several months in the clinic due to an apparent threshold effect due to the multiplicity of mitochondria alongside a complex array of protective and compensatory mechanisms ${ }^{56}$. In practice, this means that a certain amount of damage must be sustained before toxicity becomes apparent. A clear example of this is the induction of mitochondrial hepatotoxicity by fialuridine, which caused several deaths during clinical trials ${ }^{57-59}$. Toxicity was only induced following 13 weeks of exposure due to the inhibition of mitochondrial DNA replication. To replicate long-term exposure in vitro, the HepaRG model is an appropriate choice for TIER 2 mitotoxicity testing to assess chronic drug exposure-effect on mitochondrial function independent of hepatocyte cell death. Research has demonstrated that these cells are compatible with the metabolic modification assay under short exposure times $(2-24 h)^{60}$. Furthermore, the ability of this model to detect delayed mitochondrial toxicity has been shown for a panel of mitotoxicants with varied mitochondrial targets, including mitochondrial DNA, mitochondrial protein synthesis and fatty acid oxidation ${ }^{61,62}$. Importantly, this model enabled the pathway of mitochondrial toxicity to be delineated at concentrations in the region of drug exposure (Cmax, Css) in clinical practice. The metabolic modification assay (glu-gal) has also been shown to be applicable for cases of delayed toxicity in the HepaRG model. Importantly, it was able to identify fialuridine as a mitotoxicant after 2 weeks of exposure with subsequent mechanistic studies, performed over 4

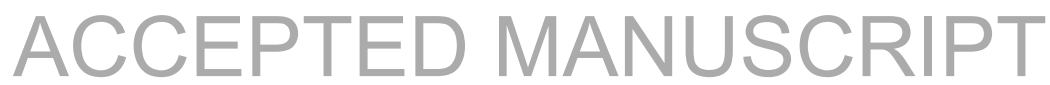


weeks, confirming the mechanism of toxicity via inhibition of mitochondrial DNA replication ${ }^{63}$. The use of this model to assess mitochondrial toxicity against a background of underlying liver pathology has also been described. In particular, steatotic HepaRG models have been developed, and used to evaluate the effects of steatosis on cellular bioenergetics and individual susceptibility to mitotoxicants ${ }^{61}$.

TIER 2 mitotoxicity testing can also encompass 3D cell systems, such as spheroids, which represent a more functionally-relevant liver system ${ }^{64}$. Work to create 3D spheroids of primary human hepatocytes (PHH) has revealed an increased sensitivity to fialuridine over extended periods, with effects beginning at 7 days and increasing over 28 days of exposure ${ }^{21}$. Such studies reveal the importance of temporal toxicodynamic events not captured in short-term assays.

Although TIER 1 and TIER 2 testing has developed to improve the detectability of mitotoxicants, these results must always be viewed as identification of chemical liabilities in the absence of quantitative IVIVE extrapolation and PKPD clinical readout. Traditionally, it has been difficult to bridge from in vitro studies to humans using animal models of mitotoxicity. Specifically, in vivo studies do not adequately represent the clinical situation as they are often performed in young and healthy in-bred rodent models which have therefore a reduced sensitivity to mitotoxicants ${ }^{65}$. As such, TIER 3 systems for evaluating mitotoxicity remain less well-defined. However, one successful example was the susceptibility of the chimeric TK-NOG mouse with humanised liver to fialuridine-induced hepatotoxicity, at doses approximately ten times the therapeutic exposure, which presented with clinical and pathological features in line with the DILI observed in humans ${ }^{66}$.

It is clear that the current DILI roadmap for mitotoxicity testing can identify certain mitochondrial liabilities. However, there is still no clear path to predicting potential risk in patients. In silico modelling is beginning to provide systems with which to bridge this translation by taking into account patient-specific factors, for example, individual health or disease state, in order to assess impact on mitochondrial toxicity. One example of such modelling is found in DILI-sym ${ }^{\circledR}$, i.e. mitoSYM ${ }^{\circledR}$, which has 
been developed to aid the mechanistic understanding of clinical hepatotoxicity and was used successfully to determine retrospectively the role of mitochondria in tolvaptan hepatotoxicity and most recently macrolide antibiotics and TAK-875 $5^{67-70}$. Overall, the last 10 years have seen significant advances in the development of models and test systems to identify mitochondrial toxicity. Practically, it is recommended that they are deployed in preclinical safety testing in a step-wise approach; TIER 1 is suitable for the risk assessment of many compounds, whilst TIER 2 and subsequent mechanistic work incorporating temporal toxicodynamic events can be used to follow-up on selected compounds of interest. TIER 3 remains a work in progress which is continuing to develop as the fundamental mechanisms directing inter-individual variation and species selectivity are discovered. Until such further progress is made, TIER 3 can function as an opportunity to retrospectively assign mechanisms and understand at-risk populations once clinical signals of hepatotoxicity have been observed.

Energy metabolism and in particular, mitochondria respiration are key processes in the build-up of fatty liver deposits as the site of fatty acid oxidation, and many of the drugs known to interact with the mitochondria at the level of $\beta$-oxidation, electron transport chain or mtDNA are associated with hepatic steatosis ${ }^{71}$. Drugs implicated in hepatic steatosis affecting the electron transport chain (ETC) and $\beta$-oxidation of fatty acids is metabolically linked, such that drugs affecting ETC pathway invariably inhibit $\beta$-oxidation. The rate-limiting step for fatty acid oxidation is the mitochondrial carnitinepalmitoyl transferase- 1 system; when mitochondrial $\beta$-oxidation is severely inhibited, fatty acyl-CoA $\beta$-oxidation is impaired with increased fatty acyl-CoA and fatty acids. These fatty acids are converted into triglycerides that are believed to be hepato-protective mechanisms of importance in mitochondrial toxicity ${ }^{72}$. Further details on the assessment and mechanisms of steatosis and detection thereof are provided in section 3 of the supplementary information. 


\section{Transporters in drug- and bile acid (BA)- induced liver injury}

Drug-induced cholestatic liver disorders are among the most severe clinical manifestations of human DILI and account for almost half of the cases of drug-related hepatotoxicity ${ }^{73,74}$. These liver disorders are broad, yet are characterized by acute and chronic impaired hepatocellular secretion of bile, with accumulation of systemic BAs and their bile salts, bilirubin and cholesterol (Supplementary information).

In many cases, the disruption of BA secretion results from functional changes in the hepatobiliary transporter system. These membrane transporters facilitate uptake and efflux of endogenous substances and drugs between the basolateral, sinusoidal and apical (bile canalicular) membranes of the hepatocyte. Many drugs are also identified as inhibitors of these membrane transporters (Figure 3). The localization of membrane transporters, expression of family and sub-family members, and polarity of transporter proteins convey the highly-regulated physiological and pharmacological role in the hepatobiliary transport of BAs (supplemental text).

Many drugs inhibit the biliary excretion of conjugated bile salts, mediated by BSEP ${ }^{75}$. Trafficking of intracellular pools of membrane transporters, such as BSEP, can be swiftly recruited and inserted into membranes as a result of drugs or increases of intracellular BAs. BSEP inhibition studies have been proposed for the assessment of human DILI and the cholestatic potential of drugs ${ }^{76-78}$. These inhibition studies are often implemented as non-cell-based screening TIER 1 assays to rank compounds that inhibit BSEP alongside other hepatobiliary transporters. Nevertheless, there are false positives and false negatives identified when solely screening for liabilities associated with BSEP inhibition ${ }^{77,78}$, as not all cholestatic drugs inhibit BSEP and detection of BSEP inhibition will be dependent to some extent on the methodologies and test systems employed ${ }^{31}$. A further complexity are drugs (e.g. captopril and cimetidine) known to cause cholestasis without apparent interaction with BA transporters ${ }^{79}$ or transporters associated with BA-independent flow. Therefore, correct classification of drugs on the basis of liver-induced severity may also explain some conflicting results. Using the FDA

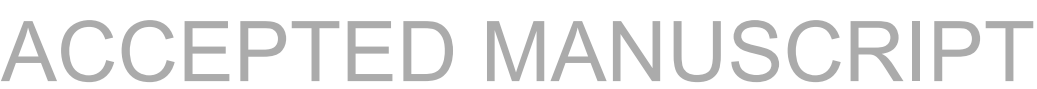


DILI severity assessment, published BSEP inhibition datasets were recently re-examined, and it was concluded that the inverted vesicle assay is not predictive of the toxic potential of drugs ${ }^{80}$.

Inverse membrane vesicles expressing human transporters allow us to detect only direct interactions with drugs. The influence of cellular factors that can be critical in the occurrence of DILI can be investigated only with polarized functional hepatocytes as part of a TIER 1 testing strategy. Sandwichcultured human hepatocytes have been widely used to evaluate BA disposition; indeed over time in sandwich configuration, hepatocytes develop functional bile canalicular networks and express hepatic transport proteins on the correct membrane domains. This cell model has served widely for studies on drug-induced alterations of BA disposition using taurocholate as a model BA. Effects of drugs on canalicular versus basolateral efflux, measurements on BA accumulation in cells and bile canaliculi versus medium have been extensively analyzed ${ }^{81-86}$. However, published data sets are scarce. The sandwich-cultured human hepatocyte model has allowed a better separation of BSEP inhibitors associated with severe DILI (e.g. cyclosporine, ritonavir, troglitazone) from those with no DILI or mild DILI (e.g. rifampicin) ${ }^{78}$. Despite many studies to try and relate BSEP inhibition and DILI, there is a recognized lack of causality between drug exposure and BSEP inhibition and human DILI ${ }^{87}$.

A fraction of bile canaliculi (BC) appear irregularly dilated with conspicuous alterations of microvilli in cholestatic liver ${ }^{88}$. Cultured PHH and HepaRG hepatocytes exhibit an early response to cholestatic drugs by the deregulation of bile canaliculi dynamics through the Rho-kinase and Myosin Light Chain kinase kinase (MLCk) kinase pathways, with or without inhibition of BSEP and NTCP activities ${ }^{89}$. These effects of deregulation of bile canaliculi dynamics occur through the constriction or dilatation of bile canaliculi associated with actin cytoskeleton disruption, and the extent of these effects are time- and drug concentration-dependent. These features further support the secondary effects of enhanced basolateral excretion of BAs and down-regulation of genes related to bile transport, synthesis and detoxification ${ }^{90,91}$. 
Early BC deformation associated with deregulation of the Rho-kinase and MLCK pathways appear to represent common features induced by cholestatic drugs. Importantly, a causal relationship between drug exposure and $\mathrm{BC}$ deregulation appears evident for drugs with known clinical cholestatic or rare cases of cholestatic potential in human. Drugs recognized as cholestatic or involved in only rare cases of clinical cholestasis were found to cause dilatation or constriction of $\mathrm{BC}^{8,20,92}$. An additional drug, i.e. macitentan, which possesses a similar chemical structure to bosentan was also classified as cholestatic $^{8}$. It is noteworthy that the first case of acute liver failure associated with macitentan treatment was only recently reported, and that histology revealed chronic hepatitis associated with a process of micronodular cholestatic cirrhosis ${ }^{93}$.

Little information exists on the early changes in BA total content and profiles in human liver exposed to cholestatic drugs. Hydrophobic BAs are much less hepatotoxic in humans than in various animal species $^{85,94}$. Accordingly, GCDCA and LCA were found to be cytotoxic in in vitro only at very high concentrations ${ }^{85,95}$. PHH and HepaRG hepatocytes synthesize, conjugate and secrete BAs in vitro ${ }^{95,96}$. However, only transient cellular accumulation (if any) can be detected in hepatocytes cultured in a serum-free medium. BA production is too low and in addition, synthesis and efflux can be inhibited and compensatory secretion via the sinusoidal transporters MRP3/4 activated by cholestatic drugs ${ }^{97}$. In fact, the cells have to be incubated with exogenous BAs at physiological or higher serum concentrations to observe any cellular accumulation of BAs. In such conditions, cellular accumulation of unconjugated CDCA and DCA and their conjugates, as well as of LCA and sulfated LCA and to a lesser extent unconjugated CA and its conjugates, can be observed with cholestatic compounds after $24 \mathrm{~h}$ treatment at non-cytotoxic concentrations $\mathrm{s}^{92,98}$. These results support the likelihood of an inhibition of BA conjugation and sulphation activities; they also indicate that cellular accumulation of BAs rapidly follows impairment of $B C$ dynamics and that LCA, the most lipophilic BA, is the first to accumulate within hepatocytes after addition of cholestatic drugs. Together with $\mathrm{BC}$ dynamics, the impairment of cellular accumulation of hydrophobic BAs could represent potent biomarkers of the cholestatic potential of new compounds ${ }^{92}$ and form part of a TIER 1 testing strategy to confirm results from non-

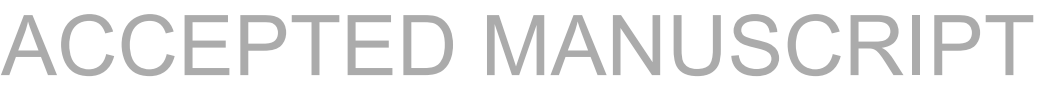


cell-based transport inhibition screening studies. Another method that has been developed for the evaluation of the cholestatic potential of drugs is based on the inhibition of urea production in sandwich-cultured human hepatocytes co-exposed to drugs and an exogenous BA mixture ${ }^{99}$. Compared to the preceding method that measures cellular accumulation of toxic BAs, this druginduced cholestatic index likely reflects BA-enhanced cellular toxicity of cholestatic drugs rather than changes in BA secretion and formation. In addition, 3D PHH spheroids have been used for predicting drug-induced changes in BA transport and hepatocellular toxicity. Highest sensitivity is seen during prolonged incubation time of the spheroids in the presence of exogenous BAs and the drug and sensitivity might be somewhat improved ${ }^{100,101}$.

The difficulties in interpreting transporter data using inverted membrane vesicles clearly warrants confirmatory studies by the use of well-characterized phenotypically-stable cell models. Sandwichcultured PHH and HepaRG hepatocytes currently appear to be the most appropriate cell models for a TIER 1 test system. They possess both physiological and anatomical features of the native hepatocyte with correctly polarized transport proteins and bile canaliculi. Of the two cell models, HepaRG hepatocytes are easier to handle and functionally relatively stable over several weeks in 2-D configuration $^{102}$. Both models can serve as a priori TIER 1 assays for early hazard identification of drugs to cause acute and even chronic changes in BA transport and secretion processes. 3-D cultures can also be obtained with PHH and HepaRG cells, and may serve as TIER 2 confirmatory test systems. In general, functions are better preserved over several weeks and co-cultures with other hepatic or nonhepatic cells can be performed. Recently, 3-D models of cholangiocytes have also been developed; they could be used to analyze extrahepatic mechanisms of toxicity induced by some compounds that cause damage to bile ducts ${ }^{103}$.

Of the TIER 1 cell-based models for the study of in situ hepatobiliary transporters, only cell-lines are readily amenable to HCA screens due to matrices required for $\mathrm{PHH}$ to maintain cell differentiation ${ }^{90,91,104-106}$ and subsequently existing methodologies for PHH are low-throughput. 
Robust quantifiable readouts required for ranking sets of compounds by use of transporter-selective probe substrates are not yet available. Most importantly, 2D PHH models do not permit time-resolved studies over extended treatment periods of several days to assess delayed onset of drug treatment without underlying changes in the constitutive phenotypic expression of DMEs and transporters. Opportunities for the use of advanced cell-based TIER 2 assays for the study of 'long-term/chronic' drug treatment on transporter function, BAs and on multiple indirect and direct targets should be more fully explored. For example, CsA and chlorpromazine exhibit concentration-dependent effects on mitochondrial membrane permeability and ER stress, respectively, which in turn may have longerterm implications on hepatobiliary transporter function. Advanced TIER 2 models, with incorporation of NPCs, would also permit more comprehensive mechanistic studies on the role of innate immune function in the initiation, adaptation and progression of changes in hepatobiliary transport function and BA secretion by drugs; the association between indirect and direct effects on the role of transporters on BAs secretion and transport, and role of the immune system are well-described ${ }^{107,108}$. Furthermore, technological advances in the development of in vitro models to explore the role of innate and adaptive immunity (TIER 3 ) in cholestasis is still required and remains an important focus for future research efforts to understand human DILI.

\section{Lysosomal perturbation}

Drug-induced phospholipidosis (PL) is the accumulation of phospholipids in cells, characterized by lamellated, membranous deposits in lysosomes. The accumulation of phospholipids can occur in any tissue, is often first observed as lung "foamy macrophages" in pre-clinical studies, and is confirmed ultrastructurally. Cationic amphiphilic drugs (CADs), such as amiodarone, perhexilene, and chlorphentermine, are typical molecular structures associated with PL. Lysosomal trapping is the mechanism by which CADs accumulate ${ }^{109}$. CADs are neutrally-charged when crossing the lysosomal membrane, but become positively charged in the acidic lysosomal compartment and thus, less membrane permeable. Once trapped in lysosomes, CADs may directly inhibit phospholipase activity 
leading to reduced ability to process phospholipids, or bind to phospholipids, resulting in a large complex that cannot be metabolized or processed ${ }^{110}$.

In the context of DILI, the consequences of PL remain the subject of debate. Some association between DILI and PL has been proposed for drugs like amiodarone and perhexilene maleate ${ }^{111}$; however, the literature and supporting data are not very clear, not confirmatory of a real association, and suggest that the primary mechanism of toxicity is more related to mitochondrial dysfunction, disrupted ATP production and fatty acid catabolism. For example, perhexilene is a carnitine palmitoyltransferase-1 (CPT-1) inhibitor with multiple effects on mitochondrial function and in vivo evidence of fatty acid metabolism inhibition ${ }^{112}$. This mechanism is more consistent with the histopathological appearance of the DILI cases that have been called "pseudo-alcoholic hepatitis," rather than a classic case of phospholipidosis. Likewise, the histologic appearance of amiodarone DILI cases suggests that PL is an independent and non-related phenomenon ${ }^{113}$. Other hypotheses have suggested that PL is a novel protective mechanism of the cell, in which CADs are sequestered in the lysosomes preventing their migration and damage to critical organelles ${ }^{114}$.

In general, the pharmaceutical discovery strategy is to lessen the propensity of candidates to induce PL. However, in the absence of other issues or toxicity, PL has not been necessarily considered a signal sufficient to halt drug development. To optimize compounds toward reduced PL propensity, various in silico tools can be used to complement a TIER I approach complemented or not by in vitro assays (e.g. LipidTox assay) or high content screening approaches to identify general PL risk, often using a standard CAD as a reference control (e.g. amiodarone) ${ }^{115,116}$; these activities can often be timed with the characterisation of the physicochemical properties of compounds described in section 3. If PL remains a concern, then it will be necessary to determine if PL occurs in vivo and at what exposure along with the identification of target organs (e.g. liver PL may present less risk than neuronal or retina $\mathrm{PL}$ ) and concurrent histopathology and ultrastructural changes. Since in vitro and in silico assays have some good predictive value in terms of risk identification but are limited in terms of risk assessment, 
the primary objectives of an in vivo evaluation are to establish a better understanding of the safety margins associated with PL (and consequently, good estimates of systemic exposures are required) and the nature of the target tissues which will determine the level of risk. These in vivo studies are typically first conducted routinely for practical reasons and since the type of molecules associated with PL usually have a high volume of distribution, dosing for more than a week is preferable. In addition to traditional histopathology evaluation, special immunohistochemical methods and biomarkers may be used to strengthen the assessment ${ }^{117,118}$. Determining reversibility of PL in toxicology studies and developing a strategy for clinical monitoring may be warranted in some situations.

\section{Reactive metabolites and oxidative stress}

Numerous experimental and clinical investigations have demonstrated that chemically reactive metabolites (CRM) are produced during the biotransformation of many pharmaceuticals, including those linked with DILI in patients $7,15,119,120$. The microsomal CYPs are dominant hepatic catalysts of drug bioactivation and responsible for the transformations of the many drugs developed before limiting bioactivation became a major objective of medicinal chemistry ${ }^{119,121,122}$. Whilst this strategy has not guaranteed safety for all newly-developed drugs, it has reduced the chemical liability of molecules with respect to DILI. Nevertheless, there remain complex challenges of managing CRM in the development of safe drugs, as exemplified by the case of fasiglifam (TAK-875) which was withdrawn from a Phase III clinical trial due to hepatotoxicity that has since been linked to protein adduction by a reactive acyl glucuronide metabolite ${ }^{123}$. As the role of CRM in DILI has been reviewed extensively ${ }^{121,122,124}$, it will not be detailed again here.

A biochemical perturbation commonly associated with, but also known to occur in the absence of, CRM formation (for example, as a consequence of mitochondrial impairment) is oxidative stress, the excessive cellular generation of reactive oxygen species (ROS) and other organic radicals that has been associated with a DILI liability ${ }^{125,126}$. Oxidative stress is typically determined through the direct quantification of ROS, assessment of the deleterious cellular consequences of ROS accumulation (i.e.

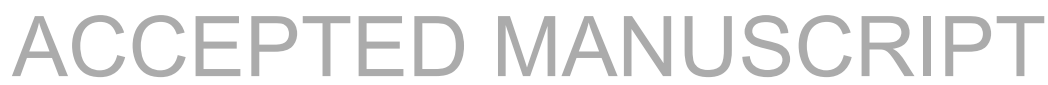


lipid peroxidation, glutathione depletion) or the evaluation of key adaptive responses (i.e. increased activity of the Nrf2 stress response pathway). In the former case, there is evidence of inadequate specificity, risking misplaced biochemical perceptions, when using some of the older and technologically less demanding ROS assays ${ }^{127-129}$, thereby promoting an unproductive proliferation of reports of oxidative stress. It is likely that the fundamental understanding of a 'probe' compound's chemistry can deliver a much more accurate assay of a $\operatorname{ROS}^{130}$ as well as more accurate biochemical understanding, leading ultimately to more accurate assessments of biological risk. Such considerations are important when probes are included in high content screening assays using simple cell models in TIER 1.

Whilst activation of the Nrf2 stress response can be determined by measuring changes in the expression level of the transcription factor and/or its conserved target genes (e.g. sulfiredoxin 1; SRXN1) in standard cell platforms, such analyses are generally more suited to low-throughput, endpoint studies. To overcome this limitation, HepG2 cells have been genetically engineered to express GFP-tagged SRXN1 and other stress response markers, thereby enabling quantitative assessment of the dynamics of the Nrf2 response in real-time using automated live cell imaging ${ }^{131,132}$. This platform represents a TIER 1 test system within the DILI roadmap (Figure 1), but could in principle be adapted to more complex models including 3D/spheroid cultures for use in Tier 2, where metabolic relevance is enhanced, as has been shown for HepG2 cultured in $3 D^{133}$. In TIER 3, the transgenic Nrf2luc reporter mouse ${ }^{134}$ has shown promise for detection of the Nrf2 response to drug-induced oxidative stress at the whole body level ${ }^{135}$. Using real-time bioluminescence imaging, localised signals were detected in the liver (acetaminophen) and kidneys (cisplatin) in vivo and ex vivo, consistent with immunohistochemical analysis that showed an elevated expression of luciferase in centrilobular hepatocytes and in tubular epithelial cells, respectively. As a low-throughput in vivo test system, the Nrf2-luc reporter mouse could be employed in bespoke mechanistic investigations (TIER 3) at later stages in preclinical drug development. In a tightly-focussed in vivo experiment, this would indicate tissue-selectivity and temporal dynamics of the physiological response to oxidative stress

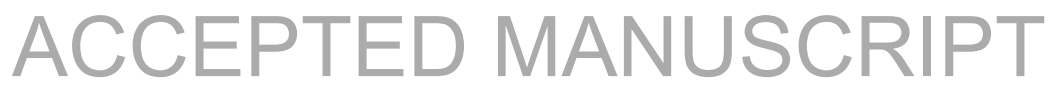


in a fully metabolically-competent model - this is relevant for human translation as it will allow a better association with dose and PK, also enabling repeat dosing and monitoring of adaptation.

Acetaminophen is typically considered to be the paradigm compound for the study of CRM and oxidative stress in hepatotoxicity ${ }^{136}$. It is well-established that cytochrome P450 (CYP)-mediated bioactivation of acetaminophen to the CRM N-acetyl-p-benzoquinoneimine results in the depletion of hepatic glutathione and the consequent accumulation of ROS and covalent modification of numerous protein targets. From the clinical perspective, it is interesting to note that delayed idiosyncratic DILI for acetaminophen has not been described, despite the fact that thousands of people take it daily for osteoarthritis. In the context of the DILI roadmap (Figure 2), it is important to note that simple cell systems such as HepG2 generally lack metabolic competence and are therefore largely incapable of reflecting the mechanisms underlying acetaminophen hepatotoxicity in vivo (extensively reviewed in $^{137}$ ), although genetic manipulation of cells to restore expression of selected CYP enzymes has been shown to improve sensitivity to acetaminophen and other selected hepatotoxicants ${ }^{11}$. In the future, as more CRM-forming drugs become eliminated from preclinical programs ${ }^{121}$, the metabolic deficiencies of simple cell systems could become less of a hindrance in early drug safety testing. Experimental confirmation of the value of a test system for examining the toxicity associated with acetaminophen and similar compounds can be gained by assessing the impact of CYP inhibition (e.g. with 1-aminobenzotriazole) on drug-induced changes in pertinent cellular readouts. For example, such an intervention was found to ameliorate the hepatotoxicity and localised reporter signal in acetaminophen-treated Nrf2-luc mice ${ }^{135}$, which is consistent with the role of CRM formation and oxidative stress in the activation of the Nrf2 stress response in this setting.

Beyond acetaminophen, a key question that remains to be addressed is whether other drugs are capable of inducing toxicologically-relevant oxidative stress in human, considering differences in daily dose (acetaminophen dosing is up to $4 \mathrm{~g}$ per day, newer drugs are typically administered $<100 \mathrm{mg}$ per 
day) and the very high concentration of the hepatic glutathione pool (5-10 mM) that must be depleted in order to allow CRM and/or ROS to accumulate to toxic levels.

\section{Endoplasmic reticulum stress}

Direct hepatotoxic drugs can induce oxidative stress in different organelles, such as endoplasmic reticulum (ER), leading to necrosis or apoptosis. Such stress will induce adaptive stress response pathways, including the ER unfolded protein response (UPR), which is initiated by three ER transmembrane proteins: IRE1 $\alpha$, PERK, and ATF6 ${ }^{138}$. Under physiological conditions, the unfolded proteins accumulated in the ER can activate these three signalling proteins either through the ERresident chaperone Bip or through direct binding to them. The activation of these proteins is important to restore ER homeostasis by increasing expression of ER chaperones and antioxidant response through the Nrf2 pathway, decreasing mRNA translation, and enhancing degradation of misfolded proteins ${ }^{139,140}$. When the activation of the UPR fails to protect survival, the cell actively pursues the proapoptotic pathway, ultimately leading to apoptotic cell death, inflammation and/or fat accumulation. This includes hyperactivation of protein kinase R-like ER kinase (PERK)/ATF6 mediated activation of C/EBP homologous protein (CHOP), IRE1-mediated activation of TRAF2, and increased $\mathrm{Ca} 2+$ release from the $\mathrm{ER}^{141}$. The precise point at which this shift from adaptation to apoptosis occurs is not certain but clearly is influenced by the degree and the duration of the ER stress.

ER stress has been associated with various drug-induced liver lesions and recently a clear link between ER stress pathway activation and drug-induced cholestasis has been reported ${ }^{92}$, where penicillinaseresistant antibiotics (flucloxacillin, cloxacillin and nafcillin) caused early cholestatic effects through the induction of ER stress.

The exact cause and effect relationship between ER stress and the mechanism of induced cell injury remains unclear. There appears to be a complex interplay between ER stress, conditions that promote 
it, and those that result from it, giving rise to a cycle in which ER stress can eventually promote inflammation, cell injury and steatosis, all of which can (subsequently) exacerbate ER stress ${ }^{142}$.

Wink et $\mathrm{al}^{131}$ have recently established a robust high-throughput imaging-based platform for the single-cell assessment of adaptive stress response pathway activation, based on a specific BAC-GFP HepG2 reporter cell line reflecting ER stress / UPR response pathways (specific biomarkers - XBP1, ATF4, BiP and CHOP). These UPR reporters have been applied together with above-mentioned Nrf2 pathway reporters to evaluate these reporter systems against a panel of $>100$ DILI compounds with different DILI risk ${ }^{132}$.

\section{The immune system in DILI}

Immune-mediated DILI is rare and the mechanism of tissue injury is believed to be a complex multicellular event. There is currently no screening strategy that can detect and eliminate candidates that may cause immune-mediated DILI. Below, we will review the evidence that implicates the innate (non-specific) and adaptive (antigen-specific) immune system in DILI at the chemical and cellular level, and critically assess what test systems might eventually be used for predicting hazard of NCEs using the DILI roadmap.

\section{Detection of drug-specific T-cells in patients with DILI}

The adaptive immune system has been implicated in DILI when there is a delayed onset. Histological investigation of liver from a patient with idiosyncratic DILI revealed an accumulation of granzyme Bsecreting T-lymphocytes alongside apoptotic hepatocytes, suggesting that T-lymphocytes participate in the adverse event ${ }^{143}$. Moreover, it has been possible to isolate drug-specific T-cells from PBMC of patients with DILI, but not drug-exposed tolerant controls. Using the lymphocyte transformation test it was possible to detect drug-responsive lymphocyte responses in approximately $50 \%$ of patients with DILI $^{144}$. Furthermore, the lymphocyte transformation test was reported to be positive in $95 \%$ of isoniazid DILI cases, whereas lymphocyte responses were not detected in patients receiving isoniazid

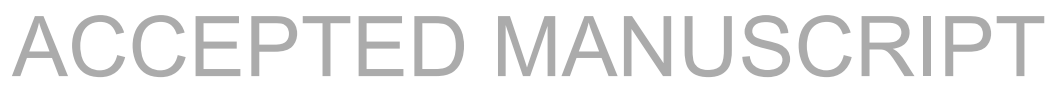


without evidence of liver damage ${ }^{145}$. Cloning T-cells from patients with isoniazid-induced DILI identified CD4+ T-cells that release an array of cytokines following drug stimulation ${ }^{146}$. A range of antidrug and autoantibodies have been identified in patients with isoniazid-induced DILI ${ }^{147}$, but not detected in tolerant patients. In recent years, flucloxacillin-, amoxicillin- and clavulanic acid-responsive T-cells were isolated from patients with DILI and characterized in terms of cellular phenotype and mechanisms of drug antigen presentation ${ }^{148-150}$. When activated with the drug, the flucloxacillinspecific T-cells killed hepatocyte-like cells expressing the relevant HLA allele in an in vitro model ${ }^{151}$. These studies are clearly beginning to define an immune basis for DILI.

For an increasing number of DILI drugs (e.g., flucloxacillin ${ }^{152}$, augmentin ${ }^{153}$, lumiracoxib ${ }^{154}$, lapatinib ${ }^{155}$, ximelagatran $^{156}$, isoniazid ${ }^{157}$, ticlopidine ${ }^{158}$, minocycline ${ }^{159}$, terbinafine $\left.{ }^{160}\right)$, genome-wide association studies have detected specific HLA alleles as important susceptibility factors. These data suggest that a highly restricted drug-derived antigen interacts with the HLA molecule to activate T-cells in susceptible patients. In fact, activation of flucloxacillin-responsive CD8+ T-cells from patients with flucloxacillin-induced liver injury has been shown to be HLA-B*57:01-restricted, which links the genetic association to the tissue injury ${ }^{148,151}$.

However, it should also be noted that the majority of individuals who carry HLA risk alleles do not develop DILI when exposed to the culprit drug, and for most HLA-associated DILI drugs, many patients that develop DILI will not carry the risk allele.

For this reason, although HLA associations represent strong evidence for an adaptive drug-specific immune response, they are not unqualified indicators of risk. There is a need to characterize the chemical (e.g., drug-modified peptides naturally eluted from HLA molecules) and immunological parameters (e.g., immune regulation) that are superimposed onto the HLA association and HLArestricted T-cell activation to determine why specific individuals develop DILI. 
Two hypotheses that are pertinent to the development of test systems for the assessment of new drugs are (1) liver injury inducing drugs activate innate signalling, and hence provide the surge of costimulatory signalling and a cytokine environment rich in inflammatory mediators, to promote the drug-specific T-lymphocyte response and (2) susceptibility relates to dysregulated expression and activity of immune regulatory pathways (cell surface receptors, Tregs, cytokines, and innate cells, including NK T-cells, neutrophils, macrophages), which are influenced by the host's genetic makeup alongside disease and environmental factors (including infections, diet, co-exposure to other drugs). Figure 4 summarizes our current understanding of the role of the adaptive immune system in druginduced liver injury, focussing on flucloxacillin reactions as a model form of HLA allele-associated immunological DILI.

\section{Involvement of innate and adaptive immune system in animal DILI models}

A limited number of animal studies show DILI involves dysregulation of innate or adaptive components of the immune system. In some cases, additional factors are required for liver injury to develop. These include microbial factors (i.e. LPS) or cytokines (in particular TNF- $\alpha^{161,162}$ ). This has been demonstrated with amiodiarone ${ }^{161}$, trovafloxacin ${ }^{162}$, diclofenac ${ }^{163,164}$ and chlorpromazine. Of these animal models, the trovafloxacin-model is the most extensively studied. However, the onset of DILI is rapid and thus does not mimic immunological DILI in humans. Trovafloxacin causes cellular stress and apoptosis, while TNF- $\alpha$ induces the influx and activation of monocytes and neutrophils in response to tissue damage ${ }^{162,165}$. Recent studies on the induction of DILI with amodiaquine demonstrated an important role for immunoregulatory T cells in conjunction with other cells, e.g. CD4, CD8 and NK1.1 cells. After 3-4 weeks of oral exposure mice developed mild DILI, which resolved after 6 weeks, possibly due to PD1+ regulatory $\mathrm{T}$ cells and also damage-controlling macrophages. Indeed, immune tolerancebreaking conditions (anti-CTLA4 in PD-1-negative mice) worsened the condition and prevented resolution of liver injury ${ }^{166-168}$. Whilst the documented studies do not currently describe a widely applicable animal model for prediction of DILI, they do illustrate our limited knowledge of the complex

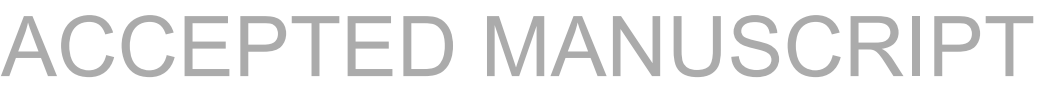


roles the innate and adaptive immune systems play in DILI. The main reason animal models cannot be used as a surrogate for human immunological drug reactions is that they do not contain fully integrated human innate and adaptive immune function. In the most recent advance in this field, HLAB*57:01-transgenic mice were generated to study HLA-linked skin reactions and a single compound, abacavir, was demonstrated to activate CD8+ $T$ cells and induce inflammation in the skin when regulatory pathways were perturbed ${ }^{169}$. Despite this, the tissue injury did not mimic that seen in humans. It is possible to consider the use of mice with humanized livers for toxicity testing purposes; however, such models have disrupted immune systems and therefore have limited application for immunological drug reactions that target liver. There is therefore a need to study human DILI in physiologically- and immunologically- relevant models using human cells, and focussing on the parameters that have been defined in patients (HLA, TCR, immune regulatory pathways).

There have been attempts to enhance the relevance of hepatocyte cell lines and co-culture systems with hepatocytes and macrophage-like cells using three-dimensional culture ${ }^{170}$. Drugs such as sulindac sulphide, chlorpromazine, diclofenac, and trovafloxacin have been shown to synergize with the cytokines TNF- $\alpha$ and IFN- $\gamma$ to kill hepatocytes in single cell culture ${ }^{162,171-173}$, indicating that it might be possible to develop relatively simple TIER 1 systems to explore innate immune signalling and druginduced hepatocyte death. Monocyte-derived hepatocyte-like cells from DILI patients have been used for causality assessment of drug-specific immune-mediated reactions ${ }^{174}$, in which in vitro toxicity testing revealed that DILI patient cells are more susceptible to culprit drugs. It will be interesting to see whether this approach can be applied to a pre-clinical setting.

\section{In vitro culture systems to explore the role of the immune system in DILI}

In order to improve the predictability of DILI risk using in vitro assays, a new cell-based assay evaluating immune and inflammatory gene expression has been developed ${ }^{175}$. The human HepaRG or HepG2 cell lines were exposed to 96 compounds and supernatants were then incubated with human promyelocytic neutrophil-derived cells (HL-60), followed by the evaluation of immune and

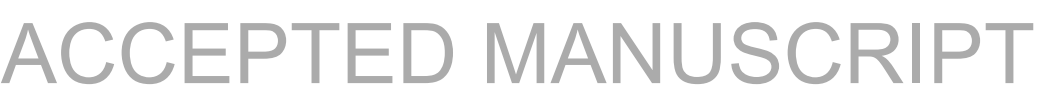


inflammatory genes. Using a combined score of S100 calcium-binding protein A9 (S100A9), IL-1B and IL-8 gene expression, the authors classified test compounds as DILI-positive and DILI-negative. To progress this assay towards a predictive test for NCEs, human drug exposure levels must be considered and whether inflammatory signals from HL-60 cells are an accurate marker for human DILI must be determined. More recently, freshly-isolated PHH have been used to characterise drug-specific signalling between the liver and innate immune cells ${ }^{176}$. Drug-treated $\mathrm{PHH}$ released damage associated molecular patterns, particularly HMGB1, in a drug- and dose-dependent manner. Furthermore, hepatocyte-conditioned media stimulated dendritic cells to secrete pro-inflammatory cytokines. However, the construction of in vitro systems to study the interface between the immune system and parenchymal cells is beset by a number of theoretical and practical obstacles. Transcriptomic analysis ${ }^{177}$ has revealed that freshly-isolated hepatocytes closely resemble "damaged" hepatocytes, which is perhaps not surprising given the conversion of intact tissue into a suspension of free cells. The theoretical concern is that such "damage" may inadvertently result in an undefined "danger signal"178 that will trigger the innate response in an uncontrolled fashion. This represents an important challenge for the engineering of organ-on-chip models which must be constructed from materials that are inert with respect to immunological activation. Ultimately, this is one of the reasons why relatively long-term 3-D hepatocyte culture ${ }^{21}$ hold promise in this area, along with iPS or adult stem cells $s^{179-184}$ for differentiation towards hepatocytes, hepatic stellate cells and cholangiocytes as well as hepatic organoids, once these models are proven to exhibit sufficient maturity. As an important aside, the conventional inclusion of supraphysiological concentrations of steroids in hepatocyte media may dampen immune responses, and this needs to be considered carefully when conducting any hepatic cell-based assays.

To study the cellular mechanisms of immune-mediated DILI in vitro, an HLA-typed PBMC bank from 1200 healthy volunteers has been established ${ }^{185}$. Furthermore, a cell culture method to assess the immunogenicity of drugs has been developed and used to explore primary T-cell responses to DILI drugs and the additional immunological parameters that determine whether formation of drug-

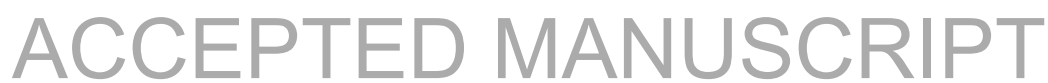


protein adducts will result in an antigen-specific T-cell response ${ }^{186-189}$. Since cross-talk between hepatocytes and immune cells is likely to be critical in determining the outcome of drug exposure, it will be necessary to develop a co-culture system using immune and iPS-derived hepatocyte-like cells from the same donors with which to begin to explore the antigenic and polarizing signals released from hepatocytes, T-cell activation and whether the activated T-cells kill hepatocytes.

In the context of the DILI roadmap (Figure 2), efforts are made to minimize exposure to chemical liabilities without biological evaluation (see section 3). This is of importance as reactive species bind covalently to selective proteins generating neo-antigens for the immune system. However, this form of assessment alone overestimates risk as many compounds that form chemical liabilities are not associated with human DILI. Furthermore, compounds such as abacavir and allopurinol that activate T-cells through unique pathways would give false negatives in screening assays for chemical liabilities or covalent binding. TIER 2 systems based on 2D or 3D microtissues consisting of liver and dendritic cells could be used to explore whether hepatocytes deliver drug metabolites and/or tissue-derived signals that result in dendritic cell activation or polarization. However, co-culture systems for screening compounds in discovery are not routinely applied for use in drug candidate screening, possibly due to the lack of guidance on how to handle the results and relate them to decision-making or the clinical situation. TIER 3 systems are proposed to explore drug-specific T-cell and antibody responses. A diagnostic toolbox of assays (e.g. lymphocyte proliferation assay, cytokine release assay, generation and characterization of antigen-specific T-cell clones) is now available to study human DILI when AEs are identified in clinical trials or when a new drug enters widespread use. Furthermore, Tcell priming assays with PBMC from HLA-typed healthy donors can be used as a retrospective investigative tool to study mechanisms of T-cell activation and to explore immunological liability of structurally-related compounds when a reaction has been seen in patients ${ }^{186,187,190}$. However, there is currently no test system available to the non-clinical scientist at present that predicts $a b$ initio the likelihood that a compound, which is clearly tolerated by $99.9 \%$ of the human population, will induce immune responses in patients. For this reason, there is no signal identified in Phase I or Phase II

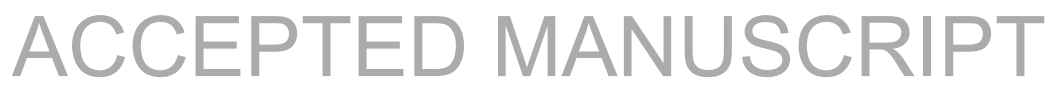


studies, or animal models which are intrinsically less heterogeneous with respect to human variables so far discovered for idiosyncratic toxicity, such as the HLA immune system. Nevertheless, we can clearly reduce some of the overall risk by eliminating compounds with particular DILI mechanisms in reproducible and well-defined models as described.

\section{Outlook}

The development of predictive models of DILI has been driven by the complementary imperatives of the pharmaceutical Industry and academic research. Industry requires serviceable predictive models, suitable for routine use in the support of drug candidate selection and decision-making that can aid a deeper understanding of the various factors that culminate in the manifestation of toxicity and can be confidently used to translate to the patient setting.

From a general perspective, whilst single cell model systems are invaluable within the tiered screening process, it is unlikely in the short-term that any single system is able to incorporate physiologicallyrelevant aspects of all of the mechanisms of DILI that have been described in the clinic and thereby demonstrate genuine predictive clinical safety of new drugs. At the same time, novel complex model systems are being used largely to further our understanding of the mechanisms involved in DILI.

The DILI roadmap (Figure 2) proposed in this perspective has therefore been developed as a guide for early drug safety evaluation, and the selection of particular test systems, with careful reference to their pharmacological and physiological relevance to the new chemical entity under consideration, before use for any toxicological investigation. Since no single currently-used model is able to recapitulate all of the mechanisms of human DILI, we believe that there exists a need for a systematic tiered approach, in which model systems of increasing biological complexity can be utilised efficiently and effectively at different points, and on different scales, in the drug discovery and development process. This tiered approach can then ultimately be connected using mathematical models. The DILI roadmap illustrates not only what can be achieved at present, but also what cannot be predicted in 
preclinical drug development at the present time, which is critical for drug regulation and public education.

A potential limitation of our strategy is that we focus predominantly on hepatocellular damage in the progression of events in human DILI. There are important examples of other forms of DILI, that require improved in vitro models for assessment of risk, and that could be introduced into the current roadmap strategy. In this Perspective, we have attempted to concentrate on the earliest chemicobiological signals that can represent perturbation in the most numerous and metabolically-active cells of the liver (the hepatocyte compartment) which can provide a biologically meaningful signal for interpretation in evaluating a range of chemical candidates. Other cells which are the primary target of compounds known to cause DILI manifestations such as sinusoidal obstructive syndrome, or vanishing bile duct syndrome - i.e. the liver sinusoidal endothelial cell (LSEC) and the biliary epithelial cell - have not been specifically examined using the roadmap approach. Nevertheless this approach would be feasible; there are established experimental protocols for isolation of these cells, and there are emerging protocols available for generating stem cell-derived LSECs and cholangiocytes ${ }^{191,192}$. Therefore, future work can apply the roadmap outlined here for establishing assays using these and indeed other non-hepatocyte liver cells and the suite of mechanistic targets discussed(e.g. mitochondrial stress, ER stress etc.) to assess the human relevance of these new cell models in screening for a range of manifestations of human DILI.

A major gap in the present armamentarium of test systems for DILI is a model which faithfully recapitulates the interaction(s) of cells of hepatic origin with cells of the immune system. The advent of more complex multicellular systems alongside MPS technologies provides much hope for the future in this respect, but such systems should be used in the first instance to understand mechanisms of immune DILI so that their pharmacological, physiological and immunological relevance can be assessed before deployment in the toxicological assessment of a new drug. Despite the many MPS and advanced tissue-based models currently available, few if any of these models appear fully 
integrated as a part of the drug discovery platforms in pharmaceutical $R \& D^{27,193}$. Several factors can be attributed to slower than expected uptake in $R \& D$, including:

1) On-going company-specific evaluations rather than a coordinated effort across industry to unify on a common platform or agreed set of platforms and clear added value ${ }^{194}$,

2) Substantial body of work required to thoroughly evaluate the physiological and pharmacological characteristics of these models and to assess their fidelity with fresh human-derived liver tissue ${ }^{195}$,

3) Curated test-set and training set of compounds to permit evidence-based step-change and improved detection of chemical insult ${ }^{196}$ and relevance to human when compared to traditional 2D hepatocellular-based culture models.

4) Confidence in preclinical to clinical PK predictions, for plasma and liver, to enable concentrationeffect understanding of DILI data.

5) Few data describing MPS responses to biologics.

Given that the immunogenic response to NCEs in idiosyncratic DILI, and also to biologics $176,197,198$, remains a key safety challenge, future MPS systems will necessitate bioengineering and integration of both the innate and adaptive immune biology to study mechanisms and emulate the response in hypersensitivity reactions. This must be supported by the continued development of novel safety biomarkers with mechanistic and translational value for use in drug discovery and clinical research ${ }^{199}$. Parallel valuation(s) of disease models of the liver to define changes in the safety margins of drugs in healthy tissue versus disease models must be performed.

Currently, MPS and advanced models are predominantly focused on the development of platforms for the study of chemical insult in humanized models. However, it is similarly imperative to demonstrate the predictive value of MPS and advanced models through use of animal MPS models for toxicology to establish a translational bridge from in vitro to in vivo pre-clinically. Human-relevant test systems 
that have been designed by retrospective analysis of DILI in humans are also needed for prospective risk assessment, representing a strategic pathway of "Human to molecule and back again".

Drug-induced liver injury in man is a function of the chemistry of the drug, the dose (mass \& duration) and human biological variables. The roadmap provides context of use of existing test systems to mitigate against DILI in humans. The roadmap also provides a future guide where the continuing efforts must shape the distinct and focused direction of linking technologies to build-in physiologically relevant and pharmacologically phenotype required of in vitro systems, which not only mimic human exposure to drug and metabolites during therapeutic conditions but also take into account the spectrum of therapeutic modalities and ever-changing nature of therapeutic innovations.

\section{Acknowledgements}

This work was supported by the European Community [Contract MIP-DILI-115336] under the Innovative Medicines Initiative Joint Undertaking and contribution from the European Union's Seventh Framework Programme [FP7/20072013] and EFPIA companies'. http://www.imi.europa.eu/.

We would like to thank Ms Karen Clayson for her excellent administrative role in the preparation and co-ordination of this article. 


\section{BOX 1. Role of dose and physico-chemical properties in the context of DILI}

\section{Human evidence}

1. Majority of oral drugs with reported idiosyncratic liver toxicity are administered at high clinical doses

2. Lipophilic drugs have higher DILI risk, explained by the increased promiscuity of high cLogP compounds. Carboxylic acid drugs metabolized by acyl glucuronidation have been associated with idiosyncratic, often liver toxicity

\section{Key points:}

- A low daily drug dose ( $<100 \mathrm{mg} /$ day) is a key attribute for lower DILI risk.

- Daily dose is a function of target potency, dose interval, and pharmacokinetic parameters

- The "rule of two" states that a high daily dose (>100 mg/day) and lipophilicity (LogP >3) enhance the risk of DILI.

- Daily dose, solubility, and lipophilicity are the three most important measures of compound quality from a medicinal chemistry perspective.

- Other physico-chemical parameters useful to assess DILI risk include carbon bond saturation and acid/base characteristics.

- Reactive metabolites can be risk factors for DILI due to their ability to bind cellular macromolecules and form adducts.

- Computational tools are available, such as structural alerts, metabolite and toxicophore prediction, CYP binding and inhibition, to guide medicinal chemists and toxicologists in the design of safer drugs. 


\section{BOX 2. Role of mitochondrial perturbation in DILI}

\section{Human evidence}

- Retrospective analysis showed that $50 \%$ of drugs with a black-box warning for hepatotoxicity contained a mitochondrial liability ${ }^{200}$.

- A PubMed search of "mitochondrial toxicity" + "drug induced liver injury" returns 332 publications since 2013

- Fialuridine, an example of a mitotoxicant with clear evidence in human ${ }^{57,201}$ : targets mtDNA, localised to the mitochondria by hENT1 (human-specific).

\section{Application of the tiered system}

- The HepG2 glucose/galactose (glu-gal) model is based on modification of the cellular bioenergetic phenotype and can be used to define chemical entities, which have a direct effect upon mitochondrial function via the electron transport chain ${ }^{50,53}$.

- Using the glu-gal model to simultaneously assess ATP cellular content and cell death can classify compounds as 1) mitochondrial toxicants and 2) mitotoxicants that lead to cell death. The assay can be used to screen parent compounds in TIER 1 to rank compounds in terms of mitotoxic liability ${ }^{53,54}$.

- The glu-gal model is compatible with other methods to detect mitotoxicity.

- Primary human hepatocytes lack the bioenergetic flexibility essential for differentiating mitotoxicity from non-mitochondrial toxicity, although they are still useful for detailed mechanistic studies.

- TIER 2 mitotoxicity testing encompasses models which allow for drug interactions with other biological systems, including xenobiotic metabolism and biliary transport, such as HepaRG cells or 3D models. These also allow longer incubations with increased clinical relevance. 


\section{BOX 3. Role of transporters and bile acids (BAs) in DILI}

\section{Human evidence}

- Hepatobiliary membrane transporters play a central role in the vectoral transport of BAs and secretion of cholephilic substances required for bile formation.

- Bile canalicular membrane transporters are highly specialized proteins, which secrete bile salts, bilirubin glucuronides, GSH conjugates and sulfoconjugated BAs.

- Drugs and metabolites directly and indirectly perturb hepatobiliary function, in particular bile canalicular function of the liver.

- Impaired bile formation and excretion arrests bile flow, characterized by accumulation of $\mathrm{BA}$ in the liver and systemic blood ${ }^{202}$.

- Arrest in bile flow leads to liver dysfunction, clinical cholestasis and concomitant hyperbilirubinemia with chronic impairment, leading to severe liver injuries including cirrhosis and liver failure ${ }^{203}$

- Progressive familial intrahepatic cholestasis type 2 (PFIC-2) is caused by mutations in BSEP 204

\section{Application of the tiered system}

- Hepatocellular-based models required to assess direct and indirect effects of drugs on hepatobiliary transporter function (TIER 1).

- Well-characterised physiological, pharmacological and phenotypically stable cell models required to study drugs and metabolites on transporter function, BA uptake and efflux ${ }^{205}$

- Key features of TIER 1 cell-models are well-formed bile canaliculi, polarized transporters and drug-metabolising enzymes, enabling the study of the effect of drugs and metabolites on BA uptake and efflux ${ }^{206}$

- Deregulation of bile canaliculi dynamics and cellular accumulation of hydrophobic BA appears a unifying feature of early events in drug-induced cholestasis.

- TIER 2 cell models would permit study of the late onset of hepatocellular dysfunction and toxicity, or adaptation, and help facilitate the extrapolation of mechanistic findings associated with hepatobiliary transport and secretion.

- TIER 3 advanced test systems would permit the study of multicellular biological interactions, including role of innate and adaptive immune function on hepatobiliary BA transport. 


\section{BOX 4. The role of chemically reactive metabolites (CRM) and oxidative stress in DILI}

\section{Human evidence:}

- Significant evidence indicates that reactive metabolites are formed from drugs known to cause hepatotoxicity. The best exemplar from clinical and preclinical studies is acetaminophen.

- The molecular signatures of drug-induced oxidative stress have been detected in many test systems for many compounds, but the relevance of these signatures to liver damage in humans, remains to be determined.

\section{Application of the tiered system:}

\section{Chemically reactive metabolites}

- Microsome-based assessment of CRM formation is a useful pre- TIER 1 chemical assay, although it takes no account of the crucial cellular context in which CRM are formed.

- In TIER 1, an accurate assessment of the extent, nature and impact of CRM formation cannot be derived from hepatocyte cell models that have been shown to lack adequate CYP activity such as HepG2 and iPSC-derived differentiated hepatocytes (see Table 1).

- If fresh PHH are unavailable, HepaRG cells, which contain significantly more CYP activity (particularly CYP3A4 ${ }^{207}$ ) than more basic cell lines can be used.

- In TIER 2, various novel cell-based models, such as 3D primary hepatocytes ${ }^{21}$ and 3D HepaRG cells are now being assessed to determine their metabolic competence.

- In TIER 3, humanised animal in vivo models may be used for mechanistic investigations on a case-by-case basis.

\section{Oxidative stress}

- In TIER 1, ROS formation can be quantified with fluorescent probes as part of a highcontent screening strategy in simple cell systems such as HepG2 or PHH.

- Genetically-modified HepG2 cells expressing GFP-tagged SRXN1 $1^{131,132}$ can be used in TIER 1 to monitor adaptive responses to oxidative stress in higher throughput using automated fluorescence microscopy.

- In TIER 2, 3D models can enable more complex biological variables, such as xenobiotic metabolism and biliary transport, and longer incubations can be used.

- In TIER 3, the transgenic Nrf2-luc reporter mouse ${ }^{135}$ can be used for investigations in a whole body context. 
BOX 5. Role of endoplasmic reticulum (ER) stress in DILI

\section{Human evidence?}

- Elevated ER stress markers reported in human liver cell lines (e.g. HepG2, HuH7, HepaRG), primary human hepatocytes and human liver slices (e.g. ${ }^{208}$ ) following exposure to several drugs, including acetaminophen, diclofenac, clozapine and efavirenz.

- ER stress studied to a far lesser extent than other mechanisms in DILI.

- More work is required to understand if ER stress is a direct causative mechanism for certain forms of human DILI, or merely a consequence of other perturbations that are more closely related to the mechanism of hepatotoxicity.

\section{Application of the tiered system}

- In tier 1, HepG2 cells can be used to examine the potential for compounds to cause ER stress, through measurement and imaging of components of UPR pathways ${ }^{131}$, and the use of UPR inhibitors to examine the effects on drug-induced toxicity.

- In tier 2, human liver slices have been used to demonstrate an UPR response to hepatotoxic drugs.

- In tier 3, western blotting and tissue staining can be used to assess the role of ER stress in the liver in vivo. Circulating biomarkers of the ER stress response will need to be identified for human translation. 


\section{BOX 6. Role of the immune system in DILI}

\section{Human evidence}

- Histological evaluation reveals innate immune cell infiltration in liver sections taken during transplantation or liver biopsy of patients with DILI

- Human evidence to support the idea that the adaptive immune system is involved in DILI:

- HLA association

- Presence of T cells in liver biopsies (flucloxacillin, sulfasalazine)

- Detection of anti-drug and auto-antibodies (isoniazid)

- Isolation of drug-specific T cells (flucloxacillin, isoniazid, amoxicillin, clavulanic acid)

\section{Application of the tiered system}

- Currently no validated assays available in TIER 1 or TIER 2 to assess the role of the innate or adaptive immune system in DILI.

- TIER 1 assays are being developed to study the release of mediators direct from the hepatocyte that have the potential to activate and recruit innate immune cells such as DAMPs. HepG2 GFPreporters for NFkB signalling (RelA and ICAM1) have been established to monitor the effect of compounds on disturbance of cytokine signalling ${ }^{172}$.

- TIER 2 assays being developed allow the investigation of cell-cell communication through direct contact or via soluble mediators. Attempts are in progress to integrate innate immune cells within 2D or 3D liver microtissues.

- Co-culture of liver cells and T cells requires an HLA-matched system. Development of stem cell technology to generate the different cell types from the liver is required before these assays can be developed.

- In TIER 3, many mouse models use supra-physiological doses and do not mimic the features of DILI in man.

- In TIER 3, in vitro T-cell assays are being developed to advance our understanding of the role of the adaptive immune system in DILI. Current applications include:

- Examination of drug-specific T cells in patients with DILI. These assays can be used to diagnose the culpable drug.

- Priming of naïve T cells. 


\section{Figures}

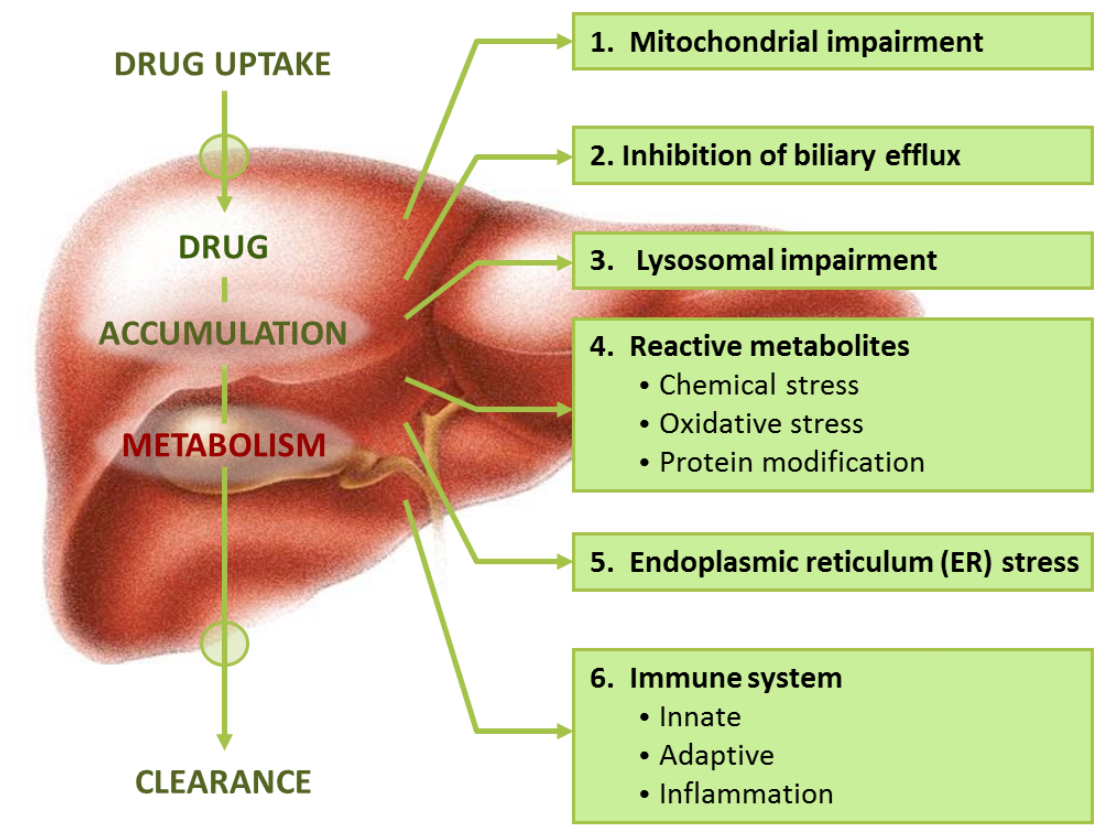

Diverse Clinical

Presentations of DILI

- Acute fatty liver with lactic acidosis

- Acute hepatic necrosis

- Acute liver failure

- Acute viral hepatitis-like liver injury

- Autoimmune-like hepatitis

- Bland cholestasis

- Cholestatic hepatitis

- Cirrhosis

- Immuno-allergic hepatitis

- Nodular regeneration

- Nonalcoholic fatty liver

- Sinusoidal obstruction syndrome

- Vanishing bile duct syndrome

Figure 1. Various chemical insults can lead to diverse clinical manifestations of DILI.

DILI can be caused by various chemical insults (1.-5.) and present as an array of different pathologies, dependent on the specific function of the liver that is impaired. Furthermore, recruitment of the immune system (6.) can result in a prolonged or altered pathological phenotype, adding further complexity to the clinical presentation of the condition. 


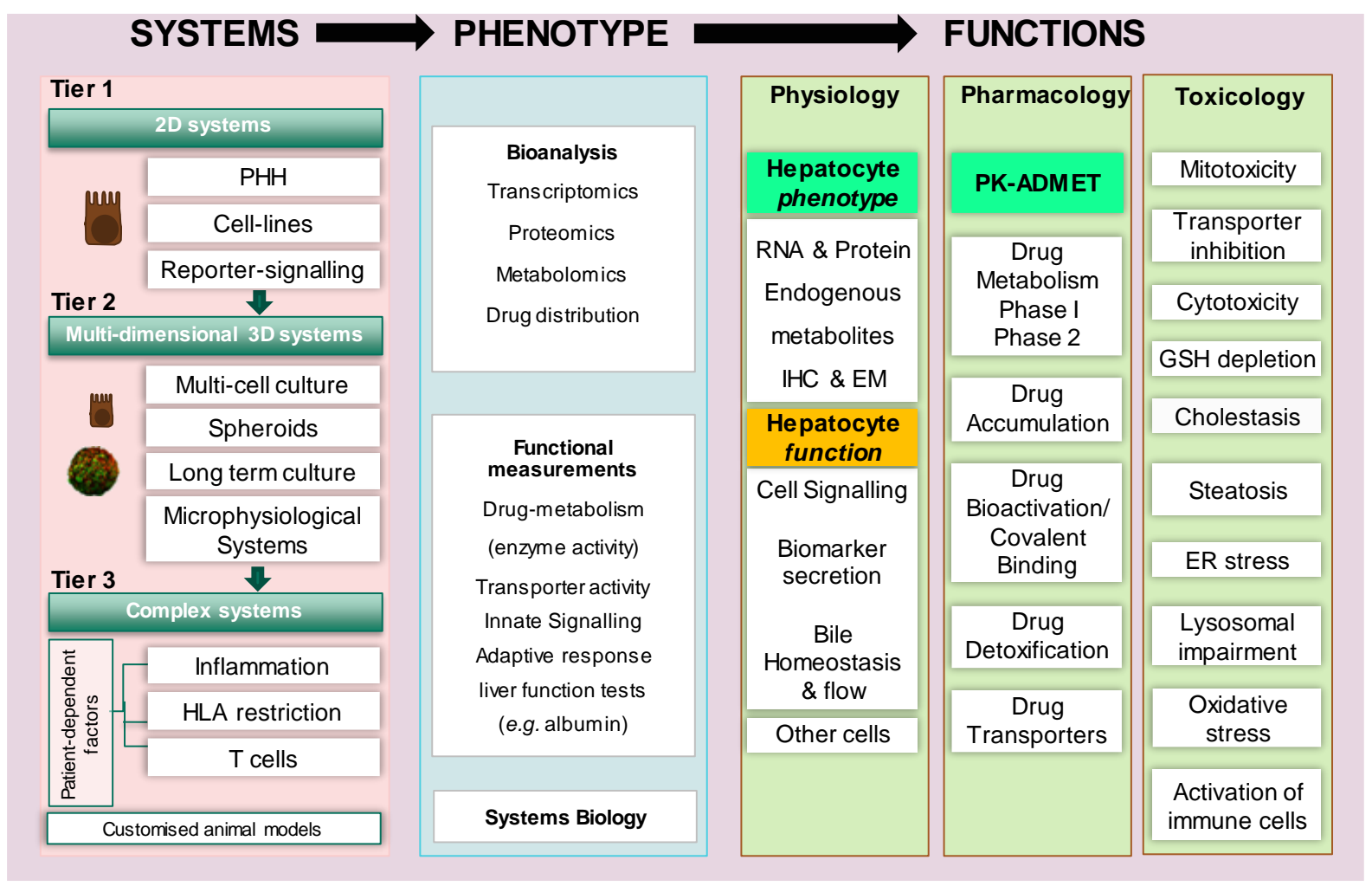

Figure 2. Roadmap for the development of 'fit-for-purpose' predictive models of human DILI

The proposed DILI Roadmap is a tier-based testing strategy incorporating present Test Systems and future expectations of advanced models for DILI testing.TIER ONE comprises single cell systems that report on immediate chemical/biological effects such as bioactivation, drug or bile acid accumulation due to transporter inhibition, mitotoxicity, and signalling associated with oxidative stress, endoplasmic reticulum stress and inflammatory signalling. TIER TWO comprises more complex systems containing liver cells in a more physiologic state, enabling assessment of the consequences of chronic drug exposure. TIER THREE comprises complex test systems in which a specific biological variable (e.g. HLA phenotype or inflammation) is introduced in a manner that can be used for both hazard identification and risk assessment related to idiosyncratic DILI. Underlying this philosophy, we believe it to be essential that the pharmacological and physiological phenotype of the test system is considered (Phenotype $\rightarrow$ Functions), before undertaking toxicological investigations, to ensure that the most appropriate methods are used to determine the potential DILI liability of a new drug. To integrate findings from different test systems and to dissect the multi-level impact of compounds, mathematical models will also be useful. 


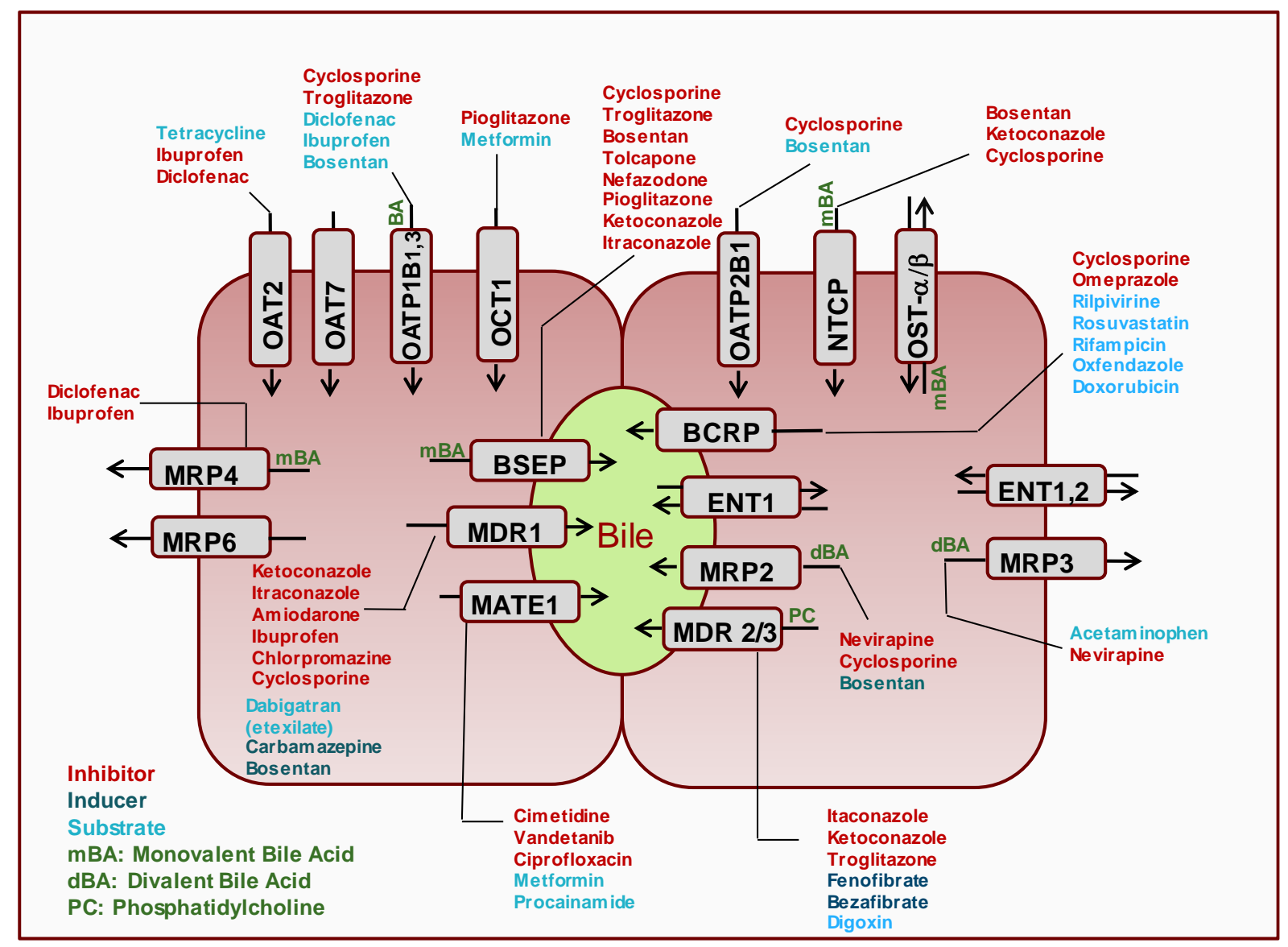

Figure 3. Hepatocyte couplet illustrating the basolateral and canalicular location of transport

proteins. Bile acids (BAs): Unconjugated BA species (BA); monovalent BA (mBA), BA-G

(glucuronidated BAs); BA-S (Sulpho and sulpho-conjugated BAs). Examples of different classes of drug substrates (blue), inhibitors (red) and inducers (purple) across multiple transporters are given. Some drugs are both substrates and inhibitors of transporter proteins depending on the affinity of respective drugs. Selectivity of transporters for the different monovalent, divalent and conjugated forms of BAs (green) across the basolateral and apical membranes illustrates the multiplicity of transporters involved in bile uptake and efflux. The heterodimeric organic solute transporter OST $\alpha /$ OST $\beta$ an efflux transporter, but also bidirectional transporter for some organic anions. Some amphiphilic BAs passively diffuse across the basolateral membrane. Phosphatidylcholine (PC) is a physiologically important substrate for MDR3 and its inhibition may play an important role in cholestasis and vanishing bile duct syndrome (VBDS). The role of taurocholic acid (TCA) in micelles has an important role on MDR3 activity. See supplementary information for transporter protein homology, function and drug interactions. 


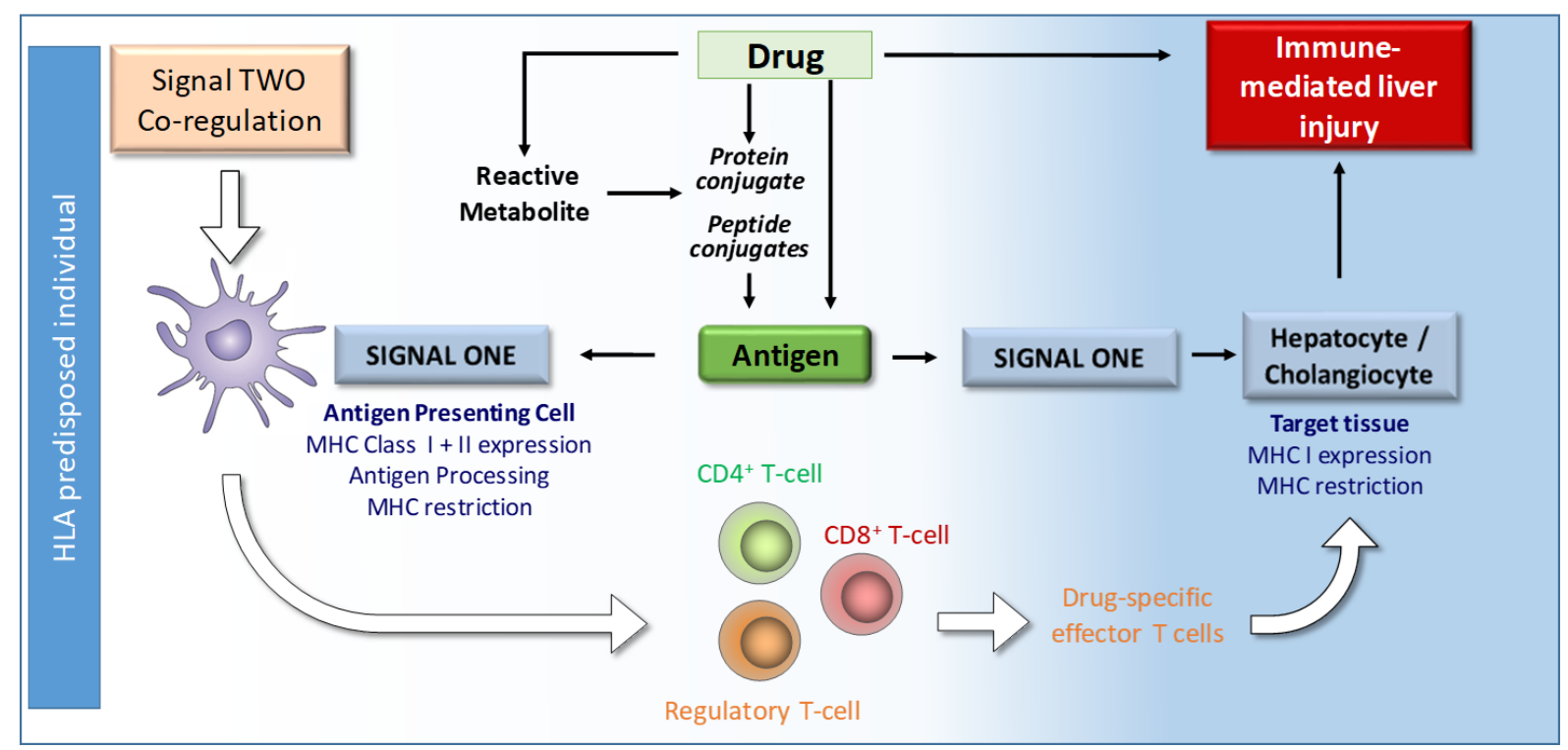

Figure 4. The role of the adaptive immune system in drug-induced liver injury

(A) Several thousand HLA allelic variants encode human MHC molecules. MHC molecules expressed on the surface of antigen-presenting cells are responsible for presentation of processed peptides to T-cells. Several forms of drug-induced liver injury have been shown to be strongly associated with expression of a single HLA. To activate T-cells, the drug must associate MHC molecules expressed on the surface of antigen-presenting cells, the peptide bound to within the MHC peptide-binding groove and T-cell receptors. To do this, drugs bind directly via a labile readily reversible interaction. Alternatively, drugs and drug metabolites act as haptens and bind covalently to protein. The resultant adduct is taken up by antigen-presenting cells and processed. The derived peptides then associate with MHC class I or II molecules for presentation to CD8+ and CD4+ T-cells, respectively (signal 1). Antigen-presenting cells receive stress signals from damaged tissue. This leads to altered expression of co-stimulatory and/or co-inhibitory receptors that interact with cognate receptors on T-cells to control the nature of the drug-specific response (signal 2). T-cells displaying reactivity to several DILI drugs have recently been isolated from PBMCs of patients with liver injury, but not tolerant controls. Furthermore, drug-specific T-cells can kill hepatocyte-like cells expressing the MHC molecule for drug presentation. 


\section{$\underline{\text { TABLES }}$}

Table 1: Relative advantages and disadvantages of the most popular single cell hepatocyte models for industry DILI assessment, including their position in the proposed tiered testing system

\begin{tabular}{|c|c|c|}
\hline Cell Type/Tier & Advantages & Disadvantages \\
\hline HepG2/TIER 1 & $\begin{array}{l}\text { Human hepatic origin. } \\
\text { Easy to culture. } \\
\text { Inexpensive. } \\
\text { Consistent, reproducible, fast turnaround } \\
\text { assay performance for the toxicological } \\
\text { endpoint under investigation. } \\
\text { No donor variation. } \\
\text { Easily adaptable for specific assays - e.g. } \\
\text { mitotoxicity assay, high-content screening. } \\
\text { Can be cultured in 3D. } \\
\text { and toxigenomics } \\
\text { Large, publicly-available datasets for } \\
\text { assessment. Popular for use in HTS approaches }\end{array}$ & $\begin{array}{l}\text { Cancer-derived. } \\
\text { Many clonal variants exist with } \\
\text { different cellular characteristics. } \\
\text { Relative lack in expression of } \\
\text { drug metabolizing enzymes and } \\
\text { transporters. Majority of genes } \\
\text { expressed in HepG2 cells are also } \\
\text { expressed in primary } \\
\text { hepatocytes, yet } 30 \% \text { of the gene } \\
\text { expression profiles are unique to } \\
\text { HepG2 }{ }^{209} \text {. }\end{array}$ \\
\hline TIER 1 or TIER 2 & $\begin{array}{l}\text { Human hepatic origin. Relatively } \\
\text { straightforward to culture. } \\
\text { More consistent than primary hepatocytes. }\end{array}$ & $\begin{array}{l}\text { Cancer cell. } \\
\text { More costly and time- } \\
\text { consuming to culture than } \\
\text { HepG2. }\end{array}$ \\
\hline
\end{tabular}




\begin{tabular}{|c|c|c|}
\hline & $\begin{array}{l}\text { Some defined Phase } 1 \text { activity (especially P450- } \\
\text { 3A4). After exposure to CYP inducers CYP450s } \\
\text { and transporter protein activities are induced } \\
\text { making them amenable for the studies of } \\
\text { ADMET mechanisms and use of gene } \\
\text { expression profiles }{ }^{209,210} \text {. Functional bile } \\
\text { canaliculi. } \\
\text { Can be cultured in 3D. }\end{array}$ & $\begin{array}{l}\text { Requires proprietary media for } \\
\text { stable phenotype }\end{array}$ \\
\hline $\begin{array}{l}\text { Human Primary } \\
\text { Hepatocytes }\end{array}$ & $\begin{array}{l}\text { The closest approximation to a liver-resident } \\
\text { hepatocyte. More metabolic activity than any } \\
\text { other liver cell model in short term cultures. } \\
\text { Can be cultured in 3D. Donor-dependent } \\
\text { metabolic phenotype allows refined risk } \\
\text { assessment. }\end{array}$ & $\begin{array}{l}\text { Loses metabolic activity rapidly } \\
\text { during culture. Variability in } \\
\text { phenotype between donors. } \\
\text { Greater cost vs cell lines. } \\
\text { Exhaustible cell supply. }\end{array}$ \\
\hline $\begin{array}{l}\text { Stem cell- } \\
\text { derived } \\
\text { hepatocytes / } \\
\text { hepatocyte-like } \\
\text { cells/ }\end{array}$ & $\begin{array}{l}\text { Reproduces many hepatocyte functions } \\
\text { Possibility of deriving hepatocyte-like cells and } \\
\text { other liver cell types from the same human } \\
\text { donor } \\
\text { Patient specific genotype/phenotype (e.g. } \\
\text { derived from DILI patients). No need to isolate } \\
\text { liver tissue per se. } \\
\text { Large-scale investment in this area. }\end{array}$ & $\begin{array}{l}\text { Expensive } \\
\text { Not as reproducible as other } \\
\text { liver cell models } \\
\text { Relative cell immaturity } \\
\text { Lack of available robust } \\
\text { differentiation protocols } \\
\text { available - different protocols } \\
\text { led to high variations in }\end{array}$ \\
\hline
\end{tabular}




\begin{tabular}{|c|c|c|}
\hline & & $\begin{array}{l}\text { transformed hepatocyte like } \\
\text { cells } \\
\text { Requires further } \\
\text { characterisation/ validation }\end{array}$ \\
\hline Liver slices & $\begin{array}{l}\text { Maintains the architecture and } \\
\text { microenvironment (cell-cell interactions) of } \\
\text { fresh tissue and therefore highly relevant to } \\
\text { the in vivo situation. } \\
\text { Good correlates of ex vivo transcriptomic } \\
\text { profiles with in vivo tissue. Coordinated } \\
\text { regulation of CYP and transporter proteins in } \\
\text { human tissue }{ }^{211} \text {. Liver function test remain } \\
\text { stable }{ }^{211,212} \text {. Recent technology developments } \\
\text { permit maintenance for 6-days }{ }^{213} \text {. }\end{array}$ & $\begin{array}{l}\text { Expression of CYP enzymes } \\
\text { remains stable for only } 24 \mathrm{~h} \text {. } \\
\text { Limited availability of human } \\
\text { fresh tissue. } \\
\text { Not amenable for HTS drug } \\
\text { screening. }\end{array}$ \\
\hline
\end{tabular}


Ostapowicz, G. et al. Results of a prospective study of acute liver failure at 17 tertiary care centers in the United States. Annals of internal medicine 137, 947-954, doi:10.7326/00034819-137-12-200212170-00007 (2002).

Atienzar, F. A. et al. Key Challenges and Opportunities Associated with the Use of In Vitro Models to Detect Human DILI: Integrated Risk Assessment and Mitigation Plans. BioMed research international 2016, 9737920, doi:10.1155/2016/9737920 (2016).

Fung, M. Evaluation of the characteristics of safety withdrawal of prescription drugs from worldwide pharmaceutical markets-1960 to 1999. Drug Inf J 35, 293-317, doi:Doi 10.1177/009286150103500134 (2001). Chen, M. et al. FDA-approved drug labeling for the study of drug-induced liver injury. Drug discovery today 16, 697-703, doi:10.1016/j.drudis.2011.05.007 (2011).

5 Thakkar, S. et al. The Liver Toxicity Knowledge Base (LKTB) and drug-induced liver injury (DILI) classification for assessment of human liver injury. Expert Rev Gastroenterol Hepatol 12, 31-38, doi:10.1080/17474124.2018.1383154 (2018). Fontana, R. J. et al. Drug-Induced Liver Injury Network (DILIN) prospective study: rationale, design and conduct. Drug safety 32, 55-68, doi:10.2165/00002018-200932010-00005 (2009). Dragovic, S. et al. Evidence-based selection of training compounds for use in the mechanismbased integrated prediction of drug-induced liver injury in man. Archives of toxicology 90, 2979-3003, doi:10.1007/s00204-016-1845-1 (2016).

Burbank, M. G. et al. From the Cover: Mechanisticlnsights in Cytotoxic and Cholestatic Potential of the Endothelial Receptor Antagonists Using HepaRG Cells. Toxicol Sci 157, 451464, doi:10.1093/toxsci/kfx062 (2017).

9 Thompson, R. A. et al. Risk assessment and mitigation strategies for reactive metabolites in drug discovery and development. Chem Biol Interact 192, 65-71, doi:10.1016/j.cbi.2010.11.002 (2011).

10 Chen, M. et al. A testing strategy to predict risk for drug-induced liver injury in humans using high-content screen assays and the 'rule-of-two' model. Archives of toxicology 88, 14391449, doi:10.1007/s00204-014-1276-9 (2014). Tolosa, L. et al. Customised in vitro model to detect human metabolism-dependent idiosyncratic drug-induced liver injury. Archives of toxicology 92, 383-399, doi:10.1007/s00204-017-2036-4 (2018). Zhu, X. W., Sedykh, A. \& Liu, S. S. Hybrid in silico models for drug-induced liver injury using chemical descriptors and in vitro cell-imaging information. J Appl Toxicol 34, 281-288, doi:10.1002/jat.2879 (2014).

13 McKim, J. M., Jr. Building a tiered approach to in vitro predictive toxicity screening: a focus on assays with in vivo relevance. Comb Chem High Throughput Screen 13, 188-206, doi:10.2174/138620710790596736 (2010).

14 Dambach, D. M., Andrews, B. A. \& Moulin, F. New technologies and screening strategies for hepatotoxicity: use of in vitro models. Toxicologic pathology 33, 17-26, doi:10.1080/01926230590522284 (2005).

15 Schadt, S. et al. Minimizing DILI risk in drug discovery - A screening tool for drug candidates. Toxicology in vitro : an international journal published in association with BIBRA 30, 429-437, doi:10.1016/j.tiv.2015.09.019 (2015).

16 Persson, M., Loye, A. F., Mow, T. \& Hornberg, J. J. A high content screening assay to predict human drug-induced liver injury during drug discovery. J Pharmacol Toxicol Methods 68, 302-313, doi:10.1016/j.vascn.2013.08.001 (2013).

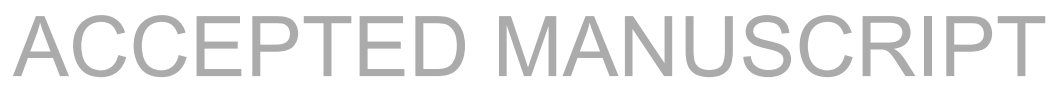


17 Tolosa, L., Gomez-Lechon, M. J. \& Donato, M. T. High-content screening technology for studying drug-induced hepatotoxicity in cell models. Archives of toxicology 89, 1007-1022, doi:10.1007/s00204-015-1503-z (2015).

18 Bell, C. C. et al. Comparison of Hepatic 2D Sandwich Cultures and 3D Spheroids for Longterm Toxicity Applications: A Multicenter Study. Toxicol Sci 162, 655-666, doi:10.1093/toxsci/kfx289 (2018).

19 Kamalian, L. et al. Acute Metabolic Switch Assay Using Glucose/Galactose Medium in HepaRG Cells to Detect Mitochondrial Toxicity. Curr Protoc Toxicol 80, e76, doi:10.1002/cptx.76 (2019).

20 Burbank, M. G. et al. Early Alterations of Bile Canaliculi Dynamics and the Rho Kinase/Myosin Light Chain Kinase Pathway Are Characteristics of Drug-Induced Intrahepatic Cholestasis. Drug Metab Dispos 44, 1780-1793, doi:10.1124/dmd.116.071373 (2016).

21 Bell, C. C. et al. Characterization of primary human hepatocyte spheroids as a model system for drug-induced liver injury, liver function and disease. Scientific reports 6, 25187, doi:10.1038/srep25187 (2016).

22 Vorrink, S. U. et al. Endogenous and xenobiotic metabolic stability of primary human hepatocytes in long-term 3D spheroid cultures revealed by a combination of targeted and untargeted metabolomics. FASEB J 31, 2696-2708, doi:10.1096/fj.201601375R (2017). Vorrink, S. U., Zhou, Y., Ingelman-Sundberg, M. \& Lauschke, V. M. Prediction of DrugInduced Hepatotoxicity Using Long-Term Stable Primary Hepatic 3D Spheroid Cultures in Chemically Defined Conditions. Toxicol Sci 163, 655-665, doi:10.1093/toxsci/kfy058 (2018).

24 Kozyra, M. et al. Human hepatic 3D spheroids as a model for steatosis and insulin resistance. Scientific reports 8, 14297, doi:10.1038/s41598-018-32722-6 (2018).

25 Lauschke, V. M., Hendriks, D. F., Bell, C. C., Andersson, T. B. \& Ingelman-Sundberg, M. Novel 3D Culture Systems for Studies of Human Liver Function and Assessments of the Hepatotoxicity of Drugs and Drug Candidates. Chem Res Toxicol 29, 1936-1955, doi:10.1021/acs.chemrestox.6b00150 (2016).

26 Beckwitt, C. H. et al. Liver 'organ on a chip'. Exp Cell Res 363, 15-25, doi:10.1016/j.yexcr.2017.12.023 (2018).

27 Bhatia, S. N. \& Ingber, D. E. Microfluidic organs-on-chips. Nat Biotechno/ 32, 760-772, doi:10.1038/nbt.2989 (2014).

28 Ewart, L. et al. Navigating tissue chips from development to dissemination: A pharmaceutical industry perspective. Experimental biology and medicine (Maywood, N.J.) 242, 1579-1585, doi:10.1177/1535370217715441 (2017).

29 Jeong, C. G., Dal Negro, G., Getsios, S. \& Ekert, J. E. in Microfluidic Cell Culture Systems (eds Jeffrey T. Borenstein, Vishal Tandon, Sarah L. Tao, \& Joseph L. Charest) 121-158 (Elsevier, 2019).

30 Weaver, R. J. \& Valentin, J. P. Today's Challenges to De-Risk and Predict Drug Safety in Human "Mind-the-Gap". Toxicol Sci 167, 307-321, doi:10.1093/toxsci/kfy270 (2019).

31 Weaver, R. J. et al. Test systems in drug discovery for hazard identification and risk assessment of human drug-induced liver injury. Expert opinion on drug metabolism \& toxicology 13, 767-782, doi:10.1080/17425255.2017.1341489 (2017).

32 Horvath, P. et al. Screening out irrelevant cell-based models of disease. Nature reviews. Drug discovery 15, 751-769, doi:10.1038/nrd.2016.175 (2016).

33 Jiang, J., Wolters, J. E., van Breda, S. G., Kleinjans, J. C. \& de Kok, T. M. Development of novel tools for the in vitro investigation of drug-induced liver injury. Expert opinion on drug metabolism \& toxicology 11, 1523-1537, doi:10.1517/17425255.2015.1065814 (2015).

34 Uetrecht, J. P. New concepts in immunology relevant to idiosyncratic drug reactions: the "danger hypothesis" and innate immune system. Chem Res Toxicol 12, 387-395, doi:10.1021/tx980249i (1999).

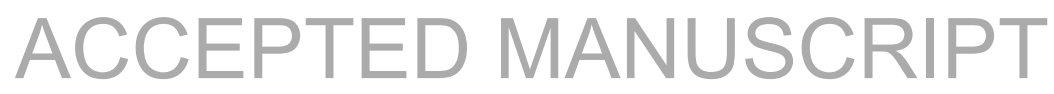



idiosyncratic hepatotoxicity. Crit Rev Toxicol 35, 325-361 (2005). lipophilicity. Drug discovery today 21, 1719-1727, doi:10.1016/j.drudis.2016.07.007 (2016). Stepan, A. F. et al. Structural alert/reactive metabolite concept as applied in medicinal chemistry to mitigate the risk of idiosyncratic drug toxicity: a perspective based on the critical examination of trends in the top 200 drugs marketed in the United States. Chem Res Toxicol 24, 1345-1410, doi:10.1021/tx200168d (2011).

38 Smith, D. A. \& Obach, R. S. Seeing through the mist: abundance versus percentage. Commentary on metabolites in safety testing. Drug Metab Dispos 33, 1409-1417, doi:10.1124/dmd.105.005041 (2005).

39 Kirchmair, J. et al. Computational prediction of metabolism: sites, products, SAR, P450 enzyme dynamics, and mechanisms. J Chem Inf Model 52, 617-648, doi:10.1021/ci200542m (2012).

40 Wenlock, M. C. \& Barton, P. In silico physicochemical parameter predictions. Mol Pharm 10, 1224-1235, doi:10.1021/mp300537k (2013).

41 Bhattacharya, S. et al. Modeling drug- and chemical-induced hepatotoxicity with systems biology approaches. Front Physiol 3, 462, doi:10.3389/fphys.2012.00462 (2012).

42 Long, A. Drug metabolism in silico - the knowledge-based expert system approach. Historical perspectives and current strategies. Drug Discov Today Technol 10, e147-153, doi:10.1016/j.ddtec.2012.10.006 (2013).

43 Blomme, E. A. \& Will, Y. Toxicology Strategies for Drug Discovery: Present and Future. Chem Res Toxicol 29, 473-504, doi:10.1021/acs.chemrestox.5b00407 (2016).

44 Bomhard, E. M. \& Herbold, B. A. Genotoxic activities of aniline and its metabolites and their relationship to the carcinogenicity of aniline in the spleen of rats. Crit Rev Toxicol 35, 783835 (2005).

45 Singh, P. K., Negi, A., Gupta, P. K., Chauhan, M. \& Kumar, R. Toxicophore exploration as a screening technology for drug design and discovery: techniques, scope and limitations. Archives of toxicology 90, 1785-1802, doi:10.1007/s00204-015-1587-5 (2016).

46 Howell, B. A. et al. In vitro to in vivo extrapolation and species response comparisons for drug-induced liver injury (DILI) using DILIsym: a mechanistic, mathematical model of DILI. Journal of pharmacokinetics and pharmacodynamics 39, 527-541, doi:10.1007/s10928-0129266-0 (2012).

47 Liu, Z. et al. Translating clinical findings into knowledge in drug safety evaluation--drug induced liver injury prediction system (DILIps). PLoS computational biology 7, e1002310, doi:10.1371/journal.pcbi.1002310 (2011).

48 Porceddu, M. et al. Prediction of liver injury induced by chemicals in human with a multiparametric assay on isolated mouse liver mitochondria. Toxicol Sci 129, 332-345, doi:10.1093/toxsci/kfs197 (2012).

49 Aleo, M. D. et al. Human drug-induced liver injury severity is highly associated with dual inhibition of liver mitochondrial function and bile salt export pump. Hepatology 60, 10151022, doi:10.1002/hep.27206 (2014).

50 Marroquin, L. D., Hynes, J., Dykens, J. A., Jamieson, J. D. \& Will, Y. Circumventing the Crabtree effect: replacing media glucose with galactose increases susceptibility of HepG2 cells to mitochondrial toxicants. Toxicol Sci 97, 539-547, doi:10.1093/toxsci/kfm052 (2007).

51 Warburg, O. On the origin of cancer cells. Science 123, 309-314, doi:10.1126/science.123.3191.309 (1956).

52 Hynes, J. et al. A high-throughput dual parameter assay for assessing drug-induced mitochondrial dysfunction provides additional predictivity over two established mitochondrial toxicity assays. Toxicology in vitro : an international journal published in association with BIBRA 27, 560-569, doi:10.1016/j.tiv.2012.11.002 (2013).

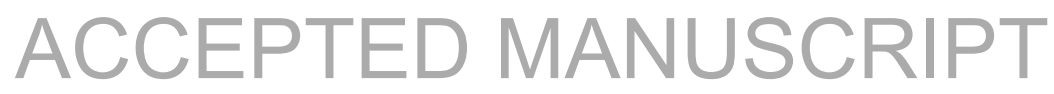


53 Kamalian, L. et al. The utility of HepG2 cells to identify direct mitochondrial dysfunction in the absence of cell death. Toxicology in vitro: an international journal published in association with BIBRA 29, 732-740, doi:10.1016/j.tiv.2015.02.011 (2015).

54 Swiss, R., Niles, A., Cali, J. J., Nadanaciva, S. \& Will, Y. Validation of a HTS-amenable assay to detect drug-induced mitochondrial toxicity in the absence and presence of cell death. Toxicology in vitro : an international journal published in association with BIBRA 27, 17891797, doi:10.1016/j.tiv.2013.05.007 (2013).

55 Eakins, J. et al. A combined in vitro approach to improve the prediction of mitochondrial toxicants. Toxicology in vitro : an international journal published in association with BIBRA 34, 161-170, doi:10.1016/j.tiv.2016.03.016 (2016).

56 Rossignol, R. et al. Mitochondrial threshold effects. Biochem J 370, 751-762, doi:10.1042/BJ20021594 (2003).

57 McKenzie, R. et al. Hepatic failure and lactic acidosis due to fialuridine (FIAU), an investigational nucleoside analogue for chronic hepatitis B. The New England journal of medicine 333, 1099-1105, doi:10.1056/NEJM199510263331702 (1995).

58 Lewis, W. et al. Fialuridine and its metabolites inhibit DNA polymerase gamma at sites of multiple adjacent analog incorporation, decrease mtDNA abundance, and cause mitochondrial structural defects in cultured hepatoblasts. P Natl Acad Sci USA 93, 35923597, doi:10.1073/pnas.93.8.3592 (1996).

59 Lee, E. W., Lai, Y., Zhang, H. \& Unadkat, J. D. Identification of the mitochondrial targeting signal of the human equilibrative nucleoside transporter 1 (hENT1): implications for interspecies differences in mitochondrial toxicity of fialuridine. J Biol Chem 281, 1670016706, doi:10.1074/jbc.M513825200 (2006).

60 Kamalian, L. et al. The utility of HepaRG cells for bioenergetic investigation and detection of drug-induced mitochondrial toxicity. Toxicology in vitro : an international journal published in association with BIBRA 53, 136-147, doi:10.1016/j.tiv.2018.08.001 (2018).

61 Le Guillou, D. et al. Drug-Induced Alterations of Mitochondrial DNA Homeostasis in Steatotic and Nonsteatotic HepaRG Cells. J Pharmacol Exp Ther 365, 711-726, doi:10.1124/jpet.117.246751 (2018).

62 Cuykx, M., Claes, L., Rodrigues, R. M., Vanhaecke, T. \& Covaci, A. Metabolomics profiling of steatosis progression in HepaRG((R)) cells using sodium valproate. Toxicology letters 286, 2230, doi:10.1016/j.toxlet.2017.12.015 (2018).

63 Jolly, C. E. et al. HepaRG cells as a physiologically and metabolically relevant in vitro model for the delayed toxicity of fialuridine via effects upon mitochondrial DNA. Archives of toxicology Status: Resubmission requested, in revision (2019).

64 Godoy, P. et al. Recent advances in 2D and 3D in vitro systems using primary hepatocytes, alternative hepatocyte sources and non-parenchymal liver cells and their use in investigating mechanisms of hepatotoxicity, cell signaling and ADME. Archives of toxicology 87, 13151530, doi:10.1007/s00204-013-1078-5 (2013).

65 Pereira, C. V., Oliveira, P. J., Will, Y. \& Nadanaciva, S. Mitochondrial bioenergetics and druginduced toxicity in a panel of mouse embryonic fibroblasts with mitochondrial DNA single nucleotide polymorphisms. Toxicol Appl Pharmacol 264, 167-181, doi:10.1016/j.taap.2012.07.030 (2012).

$66 \mathrm{Xu}, \mathrm{D}$. et al. Fialuridine induces acute liver failure in chimeric TK-NOG mice: a model for detecting hepatic drug toxicity prior to human testing. PLoS medicine 11, e1001628, doi:10.1371/journal.pmed.1001628 (2014).

67 Yang, Y. et al. MITOsym(R): A Mechanistic, Mathematical Model of Hepatocellular Respiration and Bioenergetics. Pharmaceutical research 32, 1975-1992, doi:10.1007/s11095014-1591-0 (2015).

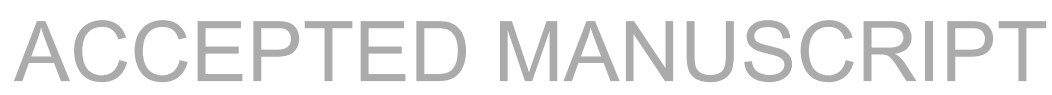


Woodhead, J. L. et al. Application of a Mechanistic Model to Evaluate Putative Mechanisms of Tolvaptan Drug-Induced Liver Injury and Identify Patient Susceptibility Factors. Toxicol Sci 155, 61-74, doi:10.1093/toxsci/kfw193 (2017). Woodhead, J. L. et al. Analyzing the Mechanisms Behind Macrolide Antibiotic-Induced Liver Injury Using Quantitative Systems Toxicology Modeling. Pharmaceutical research 36, 48, doi:10.1007/s11095-019-2582-y (2019).

70 Longo, D. M. et al. Quantitative Systems Toxicology Analysis of In Vitro Mechanistic Assays Reveals Importance of Bile Acid Accumulation and Mitochondrial Dysfunction in TAK-875Induced Liver Injury. Toxicol Sci 167, 458-467, doi:10.1093/toxsci/kfy253 (2019).

71 Simoes, I. C. M., Fontes, A., Pinton, P., Zischka, H. \& Wieckowski, M. R. Mitochondria in nonalcoholic fatty liver disease. The international journal of biochemistry \& cell biology 95, 9399, doi:10.1016/j.biocel.2017.12.019 (2018).

72 Neuschwander-Tetri, B. A. Hepatic lipotoxicity and the pathogenesis of nonalcoholic steatohepatitis: the central role of nontriglyceride fatty acid metabolites. Hepatology 52, 774-788, doi:10.1002/hep.23719 (2010).

73 Padda, M. S., Sanchez, M., Akhtar, A. J. \& Boyer, J. L. Drug-induced cholestasis. Hepatology 53, 1377-1387, doi:10.1002/hep.24229 (2011).

74 Bjornsson, E. \& Olsson, R. Outcome and prognostic markers in severe drug-induced liver disease. Hepatology 42, 481-489, doi:10.1002/hep.20800 (2005).

75 Sakurai, A., Kurata, A., Onishi, Y., Hirano, H. \& Ishikawa, T. Prediction of drug-induced intrahepatic cholestasis: in vitro screening and QSAR analysis of drugs inhibiting the human bile salt export pump. Expert opinion on drug safety 6, 71-86, doi:10.1517/14740338.6.1.71 (2007).

76 Dawson, S., Stahl, S., Paul, N., Barber, J. \& Kenna, J. G. In vitro inhibition of the bile salt export pump correlates with risk of cholestatic drug-induced liver injury in humans. Drug Metab Dispos 40, 130-138, doi:10.1124/dmd.111.040758 (2012).

77 Morgan, R. E. et al. Interference with bile salt export pump function is a susceptibility factor for human liver injury in drug development. Toxicol Sci 118, 485-500, doi:10.1093/toxsci/kfq269 (2010).

78 Pedersen, J. M. et al. Early identification of clinically relevant drug interactions with the human bile salt export pump (BSEP/ABCB11). Toxicol Sci 136, 328-343, doi:10.1093/toxsci/kft197 (2013).

79 Wang, E. J., Casciano, C. N., Clement, R. P. \& Johnson, W. W. Fluorescent substrates of sisterP-glycoprotein (BSEP) evaluated as markers of active transport and inhibition: evidence for contingent unequal binding sites. Pharmaceutical research 20, 537-544, doi:10.1023/a:1023278211849 (2003).

80 Chan, R. \& Benet, L. Z. Measures of BSEP Inhibition In Vitro Are Not Useful Predictors of DILI. Toxicol Sci 162, 499-508, doi:10.1093/toxsci/kfx284 (2018).

81 Marion, T. L., Perry, C. H., St Claire, R. L., 3rd, Yue, W. \& Brouwer, K. L. Differential disposition of chenodeoxycholic acid versus taurocholic acid in response to acute troglitazone exposure in rat hepatocytes. Toxicol Sci 120, 371-380, doi:10.1093/toxsci/kfr014 (2011).

82 Jemnitz, K., Veres, Z. \& Vereczkey, L. Contribution of high basolateral bile salt efflux to the lack of hepatotoxicity in rat in response to drugs inducing cholestasis in human. Toxicol Sci 115, 80-88, doi:10.1093/toxsci/kfq044 (2010).

83 Chiang, J. Y. Bile acids: regulation of synthesis. Journal of lipid research 50, 1955-1966, doi:10.1194/jlr.R900010-JLR200 (2009).

84 Brouwer, K. L. et al. In vitro methods to support transporter evaluation in drug discovery and development. Clin Pharmacol Ther 94, 95-112, doi:10.1038/clpt.2013.81 (2013).

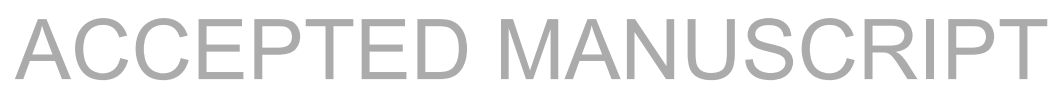


85 Woolbright, B. L. et al. Bile acid-induced necrosis in primary human hepatocytes and in patients with obstructive cholestasis. Toxicol Appl Pharmacol 283, 168-177, doi:10.1016/j.taap.2015.01.015 (2015).

86 Guo, C., LaCerte, C., Edwards, J. E., Brouwer, K. R. \& Brouwer, K. L. R. Farnesoid X Receptor Agonists Obeticholic Acid and Chenodeoxycholic Acid Increase Bile Acid Efflux in SandwichCultured Human Hepatocytes: Functional Evidence and Mechanisms. J Pharmacol Exp Ther 365, 413-421, doi:10.1124/jpet.117.246033 (2018).

87 Kenna, J. G. et al. Can Bile Salt Export Pump Inhibition Testing in Drug Discovery and Development Reduce Liver Injury Risk? An International Transporter Consortium Perspective. Clin Pharmacol Ther 104, 916-932, doi:10.1002/cpt.1222 (2018).

88 Layden, T. J., Schwarz \& Boyer, J. L. Scanning electron microscopy of the rat liver. Studies of the effect of taurolithocholate and other models of cholestasis. Gastroenterology 69, 724738 (1975).

89 Sharanek, A. et al. Rho-kinase/myosin light chain kinase pathway plays a key role in the impairment of bile canaliculi dynamics induced by cholestatic drugs. Scientific reports 6 , 24709, doi:10.1038/srep24709 (2016).

90 Antherieu, S. et al. Oxidative stress plays a major role in chlorpromazine-induced cholestasis in human HepaRG cells. Hepatology 57, 1518-1529, doi:10.1002/hep.26160 (2013).

91 Sharanek, A. et al. Different dose-dependent mechanisms are involved in early cyclosporine a-induced cholestatic effects in hepaRG cells. Toxicol Sci 141, 244-253, doi:10.1093/toxsci/kfu122 (2014).

92 Burban, A., Sharanek, A., Guguen-Guillouzo, C. \& Guillouzo, A. Endoplasmic reticulum stress precedes oxidative stress in antibiotic-induced cholestasis and cytotoxicity in human hepatocytes. Free Radic Biol Med 115, 166-178, doi:10.1016/j.freeradbiomed.2017.11.017 (2018).

93 Tran, T. T., Brinker, A. D. \& Munoz, M. Serious Liver Injury Associated with Macitentan: A Case Report. Pharmacotherapy 38, e22-e24, doi:10.1002/phar.2078 (2018).

94 Hofmann, A. F. Detoxification of lithocholic acid, a toxic bile acid: relevance to drug hepatotoxicity. Drug metabolism reviews 36, 703-722, doi:10.1081/DMR-200033475 (2004). Sharanek, A. et al. Cellular Accumulation and Toxic Effects of Bile Acids in Cyclosporine ATreated HepaRG Hepatocytes. Toxicol Sci 147, 573-587, doi:10.1093/toxsci/kfv155 (2015). Ellis, E. C. \& Nilsson, L. M. The use of human hepatocytes to investigate bile acid synthesis. Methods in molecular biology (Clifton, N.J.) 640, 417-430, doi:10.1007/978-1-60761-6887_22 (2010).

97 Zollner, G. et al. Role of nuclear bile acid receptor, FXR, in adaptive ABC transporter regulation by cholic and ursodeoxycholic acid in mouse liver, kidney and intestine. Journal of hepatology 39, 480-488, doi:10.1016/s0168-8278(03)00228-9 (2003).

98 Sharanek, A. et al. Progressive and Preferential Cellular Accumulation of Hydrophobic Bile Acids Induced by Cholestatic Drugs Is Associated with Inhibition of Their Amidation and Sulfation. Drug Metab Dispos 45, 1292-1303, doi:10.1124/dmd.117.077420 (2017).

99 Oorts, M. et al. Drug-induced cholestasis risk assessment in sandwich-cultured human hepatocytes. Toxicology in vitro: an international journal published in association with BIBRA 34, 179-186, doi:10.1016/j.tiv.2016.03.008 (2016).

100 Parmentier, C. et al. Inter-individual differences in the susceptibility of primary human hepatocytes towards drug-induced cholestasis are compound and time dependent.

Toxicology letters 295, 187-194, doi:10.1016/j.toxlet.2018.06.1069 (2018).

101 Hendriks, D. F., Fredriksson Puigvert, L., Messner, S., Mortiz, W. \& Ingelman-Sundberg, M. Hepatic 3D spheroid models for the detection and study of compounds with cholestatic liability. Scientific reports 6, 35434, doi:10.1038/srep35434 (2016).

102 Guillouzo, A. \& Guguen-Guillouzo, C. in Stem cells in toxicology and teratology (ed T.P.R.) 309-339 (John Wiley \& Sons, Inc, 2018).

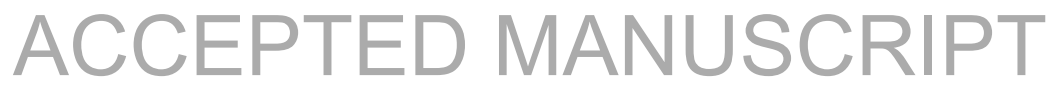


103 Loarca, L. et al. Development and characterization of cholangioids from normal and diseased human cholangiocytes as an in vitro model to study primary sclerosing cholangitis. Lab Invest 97, 1385-1396, doi:10.1038/labinvest.2017.63 (2017).

104 Swift, B., Pfeifer, N. D. \& Brouwer, K. L. Sandwich-cultured hepatocytes: an in vitro model to evaluate hepatobiliary transporter-based drug interactions and hepatotoxicity. Drug metabolism reviews 42, 446-471, doi:10.3109/03602530903491881 (2010).

105 Guguen-Guillouzo, C. \& Guillouzo, A. General review on in vitro hepatocyte models and their applications. Methods in molecular biology (Clifton, N.J.) 640, 1-40, doi:10.1007/978-160761-688-7_1 (2010).

106 Ellis, L. C., Grant, M. H., Hawksworth, G. M. \& Weaver, R. J. Quantification of biliary excretion and sinusoidal excretion of 5(6)-carboxy-2',7'-dichlorofluorescein (CDF) in cultured hepatocytes isolated from Sprague Dawley, Wistar and Mrp2-deficient Wistar (TR(-)) rats. Toxicology in vitro : an international journal published in association with BIBRA 28, 11651175, doi:10.1016/j.tiv.2014.05.010 (2014).

107 Cuperus, F. J., Claudel, T., Gautherot, J., Halilbasic, E. \& Trauner, M. The role of canalicular ABC transporters in cholestasis. Drug Metab Dispos 42, 546-560, doi:10.1124/dmd.113.056358 (2014).

108 Larson, S. P., Kovilam, O. \& Agrawal, D. K. Immunological basis in the pathogenesis of intrahepatic cholestasis of pregnancy. Expert Rev Clin Immunol 12, 39-48, doi:10.1586/1744666X.2016.1101344 (2016).

109 Alakoskela, J. M., Vitovic, P. \& Kinnunen, P. K. Screening for the drug-phospholipid interaction: correlation to phospholipidosis. ChemMedChem 4, 1224-1251, doi:10.1002/cmdc.200900052 (2009).

110 Shayman, J. A. \& Abe, A. Drug induced phospholipidosis: an acquired lysosomal storage disorder. Biochim Biophys Acta 1831, 602-611, doi:10.1016/j.bbalip.2012.08.013 (2013).

111 Schumacher, J. D. \& Guo, G. L. Mechanistic review of drug-induced steatohepatitis. Toxicol Appl Pharmacol 289, 40-47, doi:10.1016/j.taap.2015.08.022 (2015).

112 Satapathy, S. K., Kuwajima, V., Nadelson, J., Atiq, O. \& Sanyal, A. J. Drug-induced fatty liver disease: An overview of pathogenesis and management. Ann Hepatol 14, 789-806, doi:10.5604/16652681.1171749 (2015).

113 Lewis, J. H. et al. Histopathologic analysis of suspected amiodarone hepatotoxicity. Hum Pathol 21, 59-67, doi:10.1016/0046-8177(90)90076-h (1990).

114 Anderson, N. \& Borlak, J. Drug-induced phospholipidosis. FEBS Lett 580, 5533-5540, doi:10.1016/j.febslet.2006.08.061 (2006).

115 Pelletier, D. J., Gehlhaar, D., Tilloy-Ellul, A., Johnson, T. O. \& Greene, N. Evaluation of a published in silico model and construction of a novel Bayesian model for predicting phospholipidosis inducing potential. J Chem Inf Model 47, 1196-1205, doi:10.1021/ci6004542 (2007).

116 Schurdak, M. E., Vernetti, L. A., Abel, S. J. \& Thiffault, C. Adaptation of an in vitro phospholipidosis assay to an automated image analysis system. Toxicol Mech Methods 17, 77-86, doi:10.1080/15376510600860185 (2007).

117 Obert, L. A. et al. An immunohistochemical approach to differentiate hepatic lipidosis from hepatic phospholipidosis in rats. Toxicologic pathology 35, 728-734, doi:10.1080/01926230701481956 (2007).

118 Baronas, E. T., Lee, J. W., Alden, C. \& Hsieh, F. Y. Biomarkers to monitor drug-induced phospholipidosis. Toxicol Appl Pharmacol 218, 72-78, doi:10.1016/j.taap.2006.10.015 (2007).

119 Sakatis, M. Z. et al. Preclinical strategy to reduce clinical hepatotoxicity using in vitro bioactivation data for $>200$ compounds. Chem Res Toxicol 25, 2067-2082, doi:10.1021/tx300075j (2012). 
120 Thompson, R. A. et al. In vitro approach to assess the potential for risk of idiosyncratic adverse reactions caused by candidate drugs. Chem Res Toxicol 25, 1616-1632, doi:10.1021/tx300091x (2012).

121 Brink, A., Pahler, A., Funk, C., Schuler, F. \& Schadt, S. Minimizing the risk of chemically reactive metabolite formation of new drug candidates: implications for preclinical drug design. Drug discovery today 22, 751-756, doi:10.1016/j.drudis.2016.11.018 (2017).

122 Park, B. K. et al. Managing the challenge of chemically reactive metabolites in drug development. Nature reviews. Drug discovery 10, 292-306, doi:10.1038/nrd3408 (2011).

123 Otieno, M. A. et al. Mechanisms for Hepatobiliary Toxicity in Rats Treated with an Antagonist of Melanin Concentrating Hormone Receptor 1 (MCHR1). Toxicol Sci 155, 379-388, doi:10.1093/toxsci/kfw216 (2017).

124 Stachulski, A. V. et al. The generation, detection, and effects of reactive drug metabolites. Medicinal research reviews 33, 985-1080, doi:10.1002/med.21273 (2013).

125 Ramachandran, A. \& Jaeschke, H. Oxidative Stress and Acute Hepatic Injury. Curr Opin Toxicol 7, 17-21, doi:10.1016/j.cotox.2017.10.011 (2018).

126 Zhang, J. et al. Evaluation of multiple mechanism-based toxicity endpoints in primary cultured human hepatocytes for the identification of drugs with clinical hepatotoxicity: Results from 152 marketed drugs with known liver injury profiles. Chem Biol Interact 255, 311, doi:10.1016/j.cbi.2015.11.008 (2016).

127 Soh, N. Recent advances in fluorescent probes for the detection of reactive oxygen species. Anal Bioanal Chem 386, 532-543, doi:10.1007/s00216-006-0366-9 (2006).

128 Wages, P. A., Cheng, W. Y., Gibbs-Flournoy, E. \& Samet, J. M. Live-cell imaging approaches for the investigation of xenobiotic-induced oxidant stress. Biochim Biophys Acta 1860, 28022815, doi:10.1016/j.bbagen.2016.05.017 (2016).

129 Zielonka, J. et al. Global profiling of reactive oxygen and nitrogen species in biological systems: high-throughput real-time analyses. J Biol Chem 287, 2984-2995, doi:10.1074/jbc.M111.309062 (2012).

130 Zielonka, J. et al. High-throughput assays for superoxide and hydrogen peroxide: design of a screening workflow to identify inhibitors of NADPH oxidases. J Biol Chem 289, 16176-16189, doi:10.1074/jbc.M114.548693 (2014).

131 Wink, S., Hiemstra, S., Herpers, B. \& van de Water, B. High-content imaging-based BAC-GFP toxicity pathway reporters to assess chemical adversity liabilities. Archives of toxicology 91, 1367-1383, doi:10.1007/s00204-016-1781-0 (2017).

132 Wink, S., Hiemstra, S. W., Huppelschoten, S., Klip, J. E. \& van de Water, B. Dynamic imaging of adaptive stress response pathway activation for prediction of drug induced liver injury. Archives of toxicology 92, 1797-1814, doi:10.1007/s00204-018-2178-z (2018).

133 Ramaiahgari, S. C. et al. A 3D in vitro model of differentiated HepG2 cell spheroids with improved liver-like properties for repeated dose high-throughput toxicity studies. Archives of toxicology 88, 1083-1095, doi:10.1007/s00204-014-1215-9 (2014).

134 Oikawa, D., Akai, R., Tokuda, M. \& Iwawaki, T. A transgenic mouse model for monitoring oxidative stress. Scientific reports 2, 229, doi:10.1038/srep00229 (2012).

135 Forootan, S. S. et al. Real-time in vivo imaging reveals localised Nrf2 stress responses associated with direct and metabolism-dependent drug toxicity. Scientific reports 7, 16084, doi:10.1038/s41598-017-16491-2 (2017).

136 Du, K., Ramachandran, A. \& Jaeschke, H. Oxidative stress during acetaminophen hepatotoxicity: Sources, pathophysiological role and therapeutic potential. Redox Biol 10, 148-156, doi:10.1016/j.redox.2016.10.001 (2016).

137 Jaeschke, H., Xie, Y. \& McGill, M. R. Acetaminophen-induced Liver Injury: from Animal Models to Humans. J Clin Transl Hepatol 2, 153-161, doi:10.14218/JCTH.2014.00014 (2014).

138 Kim, R., Emi, M., Tanabe, K. \& Murakami, S. Role of the unfolded protein response in cell death. Apoptosis 11, 5-13, doi:10.1007/s10495-005-3088-0 (2006).

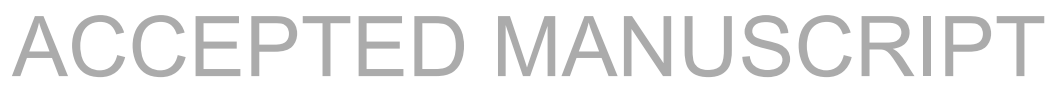


139 Hetz, C. The unfolded protein response: controlling cell fate decisions under ER stress and beyond. Nat Rev Mol Cell Biol 13, 89-102, doi:10.1038/nrm3270 (2012).

140 Oakes, S. A. \& Papa, F. R. The role of endoplasmic reticulum stress in human pathology. Annual review of pathology 10, 173-194, doi:10.1146/annurev-pathol-012513-104649 (2015).

141 Sano, R. \& Reed, J. C. ER stress-induced cell death mechanisms. Biochim Biophys Acta 1833, 3460-3470, doi:10.1016/j.bbamcr.2013.06.028 (2013).

142 Dara, L., Ji, C. \& Kaplowitz, N. The contribution of endoplasmic reticulum stress to liver diseases. Hepatology 53, 1752-1763, doi:10.1002/hep.24279 (2011).

143 Mennicke, M. et al. Fulminant liver failure after vancomycin in a sulfasalazine-induced DRESS syndrome: fatal recurrence after liver transplantation. American journal of transplantation : official journal of the American Society of Transplantation and the American Society of Transplant Surgeons 9, 2197-2202, doi:10.1111/j.1600-6143.2009.02788.x (2009).

144 Maria, V. A. \& Victorino, R. M. Diagnostic value of specific T cell reactivity to drugs in 95 cases of drug induced liver injury. Gut 41, 534-540, doi:10.1136/gut.41.4.534 (1997).

145 Warrington, R. J., Tse, K. S., Gorski, B. A., Schwenk, R. \& Sehon, A. H. Evaluation of isoniazidassociated hepatitis by immunological tests. Clinical and experimental immunology 32, 97104 (1978).

146 Usui, T. et al. From the Cover: Characterization of Isoniazid-Specific T-Cell Clones in Patients with anti-Tuberculosis Drug-Related Liver and Skin Injury. Toxicol Sci 155, 420-431, doi:10.1093/toxsci/kfw218 (2017).

147 Metushi, I. G., Sanders, C., Acute Liver Study, G., Lee, W. M. \& Uetrecht, J. Detection of antiisoniazid and anti-cytochrome P450 antibodies in patients with isoniazid-induced liver failure. Hepatology 59, 1084-1093, doi:10.1002/hep.26564 (2014).

148 Monshi, M. M. et al. Human leukocyte antigen (HLA)-B*57:01-restricted activation of drugspecific $T$ cells provides the immunological basis for flucloxacillin-induced liver injury. Hepatology 57, 727-739, doi:10.1002/hep.26077 (2013).

149 Kim, S. H. et al. Characterization of amoxicillin- and clavulanic acid-specific T cells in patients with amoxicillin-clavulanate-induced liver injury. Hepatology 62, 887-899, doi:10.1002/hep.27912 (2015).

150 Yaseen, F. S. et al. Promiscuous T-cell responses to drugs and drug-haptens. J Allergy Clin Immunol 136, 474-476 e478, doi:10.1016/j.jaci.2015.02.036 (2015).

151 Wuillemin, N. et al. HLA haplotype determines hapten or $\mathrm{p}-\mathrm{i}$ T cell reactivity to flucloxacillin. Journal of immunology (Baltimore, Md. : 1950) 190, 4956-4964, doi:10.4049/jimmunol.1202949 (2013).

152 Daly, A. K. et al. HLA-B*5701 genotype is a major determinant of drug-induced liver injury due to flucloxacillin. Nature genetics 41, 816-819, doi:10.1038/ng.379 (2009).

153 Donaldson, P. T. et al. Human leucocyte antigen class II genotype in susceptibility and resistance to co-amoxiclav-induced liver injury. Journal of hepatology 53, 1049-1053, doi:10.1016/j.jhep.2010.05.033 (2010).

154 Singer, J. B. et al. A genome-wide study identifies HLA alleles associated with lumiracoxibrelated liver injury. Nature genetics 42, 711-714, doi:10.1038/ng.632 (2010).

155 Spraggs, C. F. et al. HLA-DQA1*02:01 is a major risk factor for lapatinib-induced hepatotoxicity in women with advanced breast cancer. Journal of clinical oncology : official journal of the American Society of Clinical Oncology 29, 667-673, doi:10.1200/JCO.2010.31.3197 (2011).

156 Kindmark, A. et al. Genome-wide pharmacogenetic investigation of a hepatic adverse event without clinical signs of immunopathology suggests an underlying immune pathogenesis. The pharmacogenomics journal 8, 186-195, doi:10.1038/sj.tpj.6500458 (2008).

157 Daly, A. K. \& Day, C. P. Genetic association studies in drug-induced liver injury. Drug metabolism reviews 44, 116-126, doi:10.3109/03602532.2011.605790 (2012).

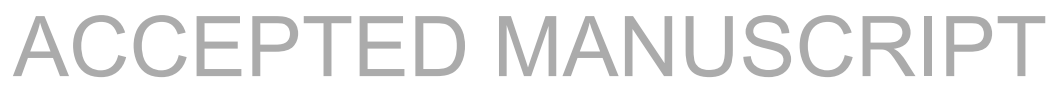


158 Kaniwa, N. \& Saito, Y. Pharmacogenomics of severe cutaneous adverse reactions and druginduced liver injury. Journal of human genetics 58, 317-326, doi:10.1038/jhg.2013.37 (2013).

159 Urban, T. J. et al. Minocycline hepatotoxicity: Clinical characterization and identification of HLA-B *35:02 as a risk factor. Journal of hepatology $67,137-144$, doi:10.1016/j.jhep.2017.03.010 (2017).

160 Nicoletti, P. et al. Association of Liver Injury From Specific Drugs, or Groups of Drugs, With Polymorphisms in HLA and Other Genes in a Genome-Wide Association Study. Gastroenterology 152, 1078-1089, doi:10.1053/j.gastro.2016.12.016 (2017).

161 Lu, J., Jones, A. D., Harkema, J. R., Roth, R. A. \& Ganey, P. E. Amiodarone exposure during modest inflammation induces idiosyncrasy-like liver injury in rats: role of tumor necrosis factor-alpha. Toxicol Sci 125, 126-133, doi:10.1093/toxsci/kfr266 (2012).

162 Shaw, P. J. et al. Trovafloxacin enhances TNF-induced inflammatory stress and cell death signaling and reduces TNF clearance in a murine model of idiosyncratic hepatotoxicity. Toxicol Sci 111, 288-301, doi:10.1093/toxsci/kfp163 (2009).

163 Deng, X. et al. Modest inflammation enhances diclofenac hepatotoxicity in rats: role of neutrophils and bacterial translocation. J Pharmacol Exp Ther 319, 1191-1199, doi:10.1124/jpet.106.110247 (2006).

164 Deng, X. et al. Gene expression profiles in livers from diclofenac-treated rats reveal intestinal bacteria-dependent and -independent pathways associated with liver injury. J Pharmacol Exp Ther 327, 634-644, doi:10.1124/jpet.108.140335 (2008).

165 Giustarini, G. et al. Trovafloxacin-Induced Liver Injury: Lack in Regulation of Inflammation by Inhibition of Nucleotide Release and Neutrophil Movement. Toxicol Sci 167, 385-396, doi:10.1093/toxsci/kfy244 (2019).

166 Mak, A. \& Uetrecht, J. The Role of CD8 T Cells in Amodiaquine-Induced Liver Injury in PD1-/Mice Cotreated with Anti-CTLA-4. Chem Res Toxicol 28, 1567-1573, doi:10.1021/acs.chemrestox.5b00137 (2015).

167 Metushi, I. G. et al. Development of a novel mouse model of amodiaquine-induced liver injury with a delayed onset. J Immunotoxicol 12, 247-260, doi:10.3109/1547691X.2014.934977 (2015).

168 Metushi, I. G., Hayes, M. A. \& Uetrecht, J. Treatment of PD-1(-/-) mice with amodiaquine and anti-CTLA4 leads to liver injury similar to idiosyncratic liver injury in patients. Hepatology 61, 1332-1342, doi:10.1002/hep.27549 (2015).

169 Cardone, M. et al. A transgenic mouse model for HLA-B*57:01-linked abacavir drug tolerance and reactivity. The Journal of clinical investigation 128, 2819-2832, doi:10.1172/JCI99321 (2018).

170 Kato, R. et al. Human hepatocarcinoma functional liver cell-4 cell line exhibits high expression of drug-metabolizing enzymes in three-dimensional culture. Biol Pharm Bull 37, 1782-1787, doi:10.1248/bpb.b14-00438 (2014).

171 Beggs, K. M. et al. Trovafloxacin-induced replication stress sensitizes HepG2 cells to tumor necrosis factor-alpha-induced cytotoxicity mediated by extracellular signal-regulated kinase and ataxia telangiectasia and Rad3-related. Toxicology 331, 35-46, doi:10.1016/j.tox.2015.03.002 (2015).

172 Fredriksson, L. et al. Diclofenac inhibits tumor necrosis factor-alpha-induced nuclear factorkappaB activation causing synergistic hepatocyte apoptosis. Hepatology 53, 2027-2041, doi:10.1002/hep.24314 (2011).

173 Zou, W., Roth, R. A., Younis, H. S., Burgoon, L. D. \& Ganey, P. E. Oxidative stress is important in the pathogenesis of liver injury induced by sulindac and lipopolysaccharide cotreatment. Toxicology 272, 32-38, doi:10.1016/j.tox.2010.03.015 (2010).

174 Benesic, A., Leitl, A. \& Gerbes, A. L. Monocyte-derived hepatocyte-like cells for causality assessment of idiosyncratic drug-induced liver injury. Gut 65, 1555-1563, doi:10.1136/gutjnl2015-309528 (2016).

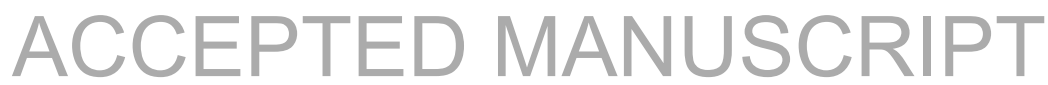


175 Oda, S., Matsuo, K., Nakajima, A. \& Yokoi, T. A novel cell-based assay for the evaluation of immune- and inflammatory-related gene expression as biomarkers for the risk assessment of drug-induced liver injury. Toxicology letters 241, 60-70, doi:10.1016/j.toxlet.2015.10.029 (2016).

176 Ogese, M. O. et al. Characterization of Drug-Specific Signaling Between Primary Human Hepatocytes and Immune Cells. Toxicol Sci 158, 76-89, doi:10.1093/toxsci/kfx069 (2017).

177 Sutherland, J. J., Jolly, R. A., Goldstein, K. M. \& Stevens, J. L. Assessing Concordance of DrugInduced Transcriptional Response in Rodent Liver and Cultured Hepatocytes. PLoS computational biology 12, e1004847, doi:10.1371/journal.pcbi.1004847 (2016).

178 Seong, S. Y. \& Matzinger, P. Hydrophobicity: an ancient damage-associated molecular pattern that initiates innate immune responses. Nature reviews. Immunology 4, 469-478, doi:10.1038/nri1372 (2004).

179 Heslop, J. A. et al. Donor-Dependent and Other Nondefined Factors have Greater Influence on the Hepatic Phenotype than the Starting Cell Type in Induced Pluripotent Stem Cell Derived Hepatocyte-Like Cells. Stem cells translational medicine 6, 1751, doi:10.1002/sctm.12187 (2017).

180 Huch, M. et al. Long-term culture of genome-stable bipotent stem cells from adult human liver. Cell 160, 299-312, doi:10.1016/j.cell.2014.11.050 (2015).

$181 \mathrm{Hu}, \mathrm{H}$. et al. Long-Term Expansion of Functional Mouse and Human Hepatocytes as 3D Organoids. Cell 175, 1591-1606 e1519, doi:10.1016/j.cell.2018.11.013 (2018).

182 Coll, M. et al. Generation of Hepatic Stellate Cells from Human Pluripotent Stem Cells Enables In Vitro Modeling of Liver Fibrosis. Cell stem cell 23, 101-113 e107, doi:10.1016/j.stem.2018.05.027 (2018).

183 Tysoe, O. C. et al. Isolation and propagation of primary human cholangiocyte organoids for the generation of bioengineered biliary tissue. Nat Protoc 14, 1884-1925, doi:10.1038/s41596-019-0168-0 (2019).

184 Peng, W. C. et al. Inflammatory Cytokine TNFalpha Promotes the Long-Term Expansion of Primary Hepatocytes in 3D Culture. Cell 175, 1607-1619 e1615, doi:10.1016/j.cell.2018.11.012 (2018).

185 Alfirevic, A. et al. In silico analysis of HLA associations with drug-induced liver injury: use of a HLA-genotyped DNA archive from healthy volunteers. Genome medicine 4, 51, doi:10.1186/gm350 (2012).

186 Faulkner, L. et al. Detection of Primary T Cell Responses to Drugs and Chemicals in HLATyped Volunteers: Implications for the Prediction of Drug Immunogenicity. Toxicol Sci 154, 416-429, doi:10.1093/toxsci/kfw177 (2016).

187 Faulkner, L. et al. The development of in vitro culture methods to characterize primary T-cell responses to drugs. Toxicol Sci 127, 150-158, doi:10.1093/toxsci/kfs080 (2012).

188 Gibson, A. et al. Negative regulation by PD-L1 during drug-specific priming of IL-22-secreting T cells and the influence of PD-1 on effector T cell function. Journal of immunology (Baltimore, Md. : 1950) 192, 2611-2621, doi:10.4049/jimmunol.1302720 (2014).

189 Gibson, A., Faulkner, L., Wood, S., Park, B. K. \& Naisbitt, D. J. Identification of drug- and drugmetabolite immune responses originating from both naive and memory T cells. J Allergy Clin Immunol 140, 578-581 e575, doi:10.1016/j.jaci.2016.11.032 (2017).

190 Usui, T. et al. Application of in Vitro T Cell Assay Using Human Leukocyte Antigen-Typed Healthy Donors for the Assessment of Drug Immunogenicity. Chem Res Toxicol 31, 165-167, doi:10.1021/acs.chemrestox.8b00030 (2018).

191 Koui, Y. et al. An In Vitro Human Liver Model by iPSC-Derived Parenchymal and Nonparenchymal Cells. Stem cell reports 9, 490-498, doi:10.1016/j.stemcr.2017.06.010 (2017).

192 Sampaziotis, F. et al. Directed differentiation of human induced pluripotent stem cells into functional cholangiocyte-like cells. Nat Protoc 12, 814-827, doi:10.1038/nprot.2017.011 (2017).

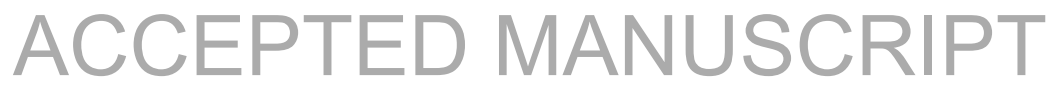


193 Holmes, A., Bonner, F. \& Jones, D. Assessing drug safety in human tissues - what are the barriers? Nature reviews. Drug discovery 14, 585-587, doi:10.1038/nrd4662 (2015).

194 Marx, U. et al. Biology-inspired microphysiological system approaches to solve the prediction dilemma of substance testing. Altex 33, 272-321, doi:10.14573/altex.1603161 (2016).

195 Cronin, M. Non-animal approaches the way forward. Report on a European Commission scientific conference held on 6-7 December, 2016, at the Egg, Brussels, Belgium. European Commission. (2017).

196 Lin, C., Ballinger, K. R. \& Khetani, S. R. The application of engineered liver tissues for novel drug discovery. Expert opinion on drug discovery 10, 519-540, doi:10.1517/17460441.2015.1032241 (2015).

197 Brennan, F. R. et al. Nonclinical safety testing of biopharmaceuticals--Addressing current challenges of these novel and emerging therapies. Regulatory toxicology and pharmacology: RTP 73, 265-275, doi:10.1016/j.yrtph.2015.07.019 (2015).

198 Dixit, R. \& Boelsterli, U. A. Healthy animals and animal models of human disease(s) in safety assessment of human pharmaceuticals, including therapeutic antibodies. Drug discovery today 12, 336-342, doi:10.1016/j.drudis.2007.02.018 (2007).

199 Matheis, K. et al. A generic operational strategy to qualify translational safety biomarkers. Drug discovery today 16, 600-608, doi:10.1016/j.drudis.2011.04.011 (2011).

200 Dykens, J. A. \& Will, Y. The significance of mitochondrial toxicity testing in drug development. Drug discovery today 12, 777-785, doi:10.1016/j.drudis.2007.07.013 (2007).

201 Lai, Y., Tse, C. M. \& Unadkat, J. D. Mitochondrial expression of the human equilibrative nucleoside transporter 1 (hENT1) results in enhanced mitochondrial toxicity of antiviral drugs. J Biol Chem 279, 4490-4497, doi:10.1074/jbc.M307938200 (2004).

202 Monte, M. J., Marin, J. J., Antelo, A. \& Vazquez-Tato, J. Bile acids: chemistry, physiology, and pathophysiology. World journal of gastroenterology 15, 804-816, doi:10.3748/wjg.15.804 (2009).

203 Fischer, S., Beuers, U., Spengler, U., Zwiebel, F. M. \& Koebe, H. G. Hepatic levels of bile acids in end-stage chronic cholestatic liver disease. Clin Chim Acta 251, 173-186, doi:10.1016/0009-8981(96)06305-x (1996).

204 Stindt, J. et al. Bile salt export pump-reactive antibodies form a polyclonal, multi-inhibitory response in antibody-induced bile salt export pump deficiency. Hepatology 63, 524-537, doi:10.1002/hep.28311 (2016).

205 Aninat, C. et al. Expression of cytochromes P450, conjugating enzymes and nuclear receptors in human hepatoma HepaRG cells. Drug Metab Dispos 34, 75-83, doi:10.1124/dmd.105.006759 (2006).

206 Guillouzo, A. et al. The human hepatoma HepaRG cells: a highly differentiated model for studies of liver metabolism and toxicity of xenobiotics. Chem Biol Interact 168, 66-73, doi:10.1016/j.cbi.2006.12.003 (2007).

207 Sison-Young, R. L. et al. Comparative Proteomic Characterization of 4 Human Liver-Derived Single Cell Culture Models Reveals Significant Variation in the Capacity for Drug Disposition, Bioactivation, and Detoxication. Toxicol Sci 147, 412-424, doi:10.1093/toxsci/kfv136 (2015).

208 Fredriksson, L. et al. Drug-induced endoplasmic reticulum and oxidative stress responses independently sensitize toward TNFalpha-mediated hepatotoxicity. Toxicol Sci 140, 144-159, doi:10.1093/toxsci/kfu072 (2014).

209 Harris, A. J., Dial, S. L. \& Casciano, D. A. Comparison of basal gene expression profiles and effects of hepatocarcinogens on gene expression in cultured primary human hepatocytes and HepG2 cells. Mutation research 549, 79-99, doi:10.1016/j.mrfmmm.2003.11.014 (2004).

210 Hart, S. N. et al. A comparison of whole genome gene expression profiles of HepaRG cells and HepG2 cells to primary human hepatocytes and human liver tissues. Drug Metab Dispos 38, 988-994, doi:10.1124/dmd.109.031831 (2010).

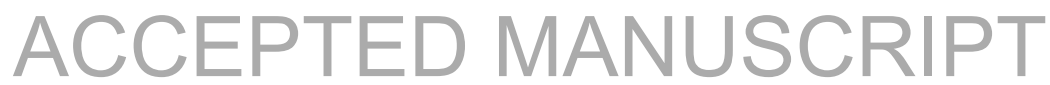


211 Olinga, P. et al. Coordinated induction of drug transporters and phase I and II metabolism in human liver slices. European journal of pharmaceutical sciences : official journal of the European Federation for Pharmaceutical Sciences 33, 380-389, doi:10.1016/j.ejps.2008.01.008 (2008).

212 Granitzny, A. et al. Maintenance of high quality rat precision cut liver slices during culture to study hepatotoxic responses: Acetaminophen as a model compound. Toxicology in vitro : an international journal published in association with BIBRA 42, 200-213, doi:10.1016/j.tiv.2017.05.001 (2017).

213 Paish, H. L. et al. A Bioreactor Technology for Modeling Fibrosis in Human and Rodent Precision-Cut Liver Slices. Hepatology 0, doi:10.1002/hep.30651 (2019). 